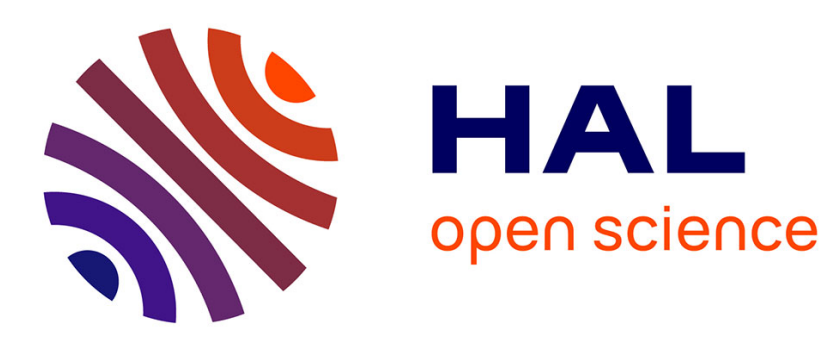

\title{
Propulsive Performance for an Oscillating Airfoil Applied to Mini Air Vehicles
}

Nikola Gavrilovic, Hesham Gaballa, Valérie Ferrand, Jean-Marc Moschetta

\section{To cite this version:}

Nikola Gavrilovic, Hesham Gaballa, Valérie Ferrand, Jean-Marc Moschetta. Propulsive Performance for an Oscillating Airfoil Applied to Mini Air Vehicles. AIAA AVIATION 2020 FORUM, Jun 2020, Virtual event, United States. pp.1-18, 10.2514/6.2020-2686 . hal-03391375

\section{HAL Id: hal-03391375 https://hal.science/hal-03391375}

Submitted on 21 Oct 2021

HAL is a multi-disciplinary open access archive for the deposit and dissemination of scientific research documents, whether they are published or not. The documents may come from teaching and research institutions in France or abroad, or from public or private research centers.
L'archive ouverte pluridisciplinaire HAL, est destinée au dépôt et à la diffusion de documents scientifiques de niveau recherche, publiés ou non, émanant des établissements d'enseignement et de recherche français ou étrangers, des laboratoires publics ou privés. 


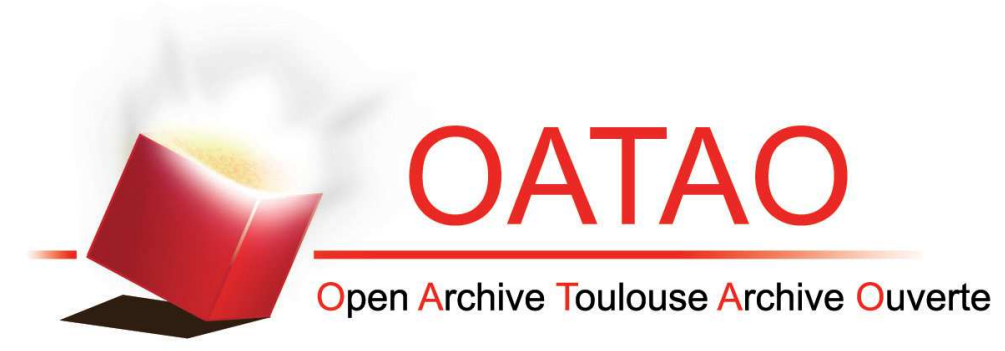

\section{Open Archive Toulouse Archive Ouverte (OATAO)}

OATAO is an open access repository that collects the work of some Toulouse researchers and makes it freely available over the web where possible.

This is an author's version published in: https://oatao.univ-toulouse.fr/28451

Official URL: https://doi.org/10.2514/6.2020-2686

\section{To cite this version :}

Gavrilovic, Nikola and Gaballa, Hesham and Ferrand, Valérie and Moschetta, Jean-Marc Propulsive Performance for an Oscillating Airfoil Applied to Mini Air Vehicles. (2020) In: AIAA AVIATION 2020 FORUM, 15 June 2020 - 19 June 2020 (Virtual event, United States).

Any correspondence concerning this service should be sent to the repository administrator: tech-oatao@listes-diff.inp-toulouse.fr 


\title{
Propulsive Performance for an Oscillating Airfoil Applied to Mini Air Vehicles
}

\author{
Nikola Gavrilović* Hesham Gaballa† Valérie Ferrand ${ }^{\ddagger}$ and Jean-Marc Moschetta ${ }^{\S}$ \\ ISAE-SUPAERO, Université de Toulouse, 31400 Toulouse, France
}

\begin{abstract}
In the present work, the optimal control to maximize the energy harvesting through a sinusoidal vertical gust profile is investigated through 2D URANS simulations and wind tunnel tests of NACA 0015 wing. The control is defined by a harmonic pitching motion of the wing, with the main objective to determine the optimal control parameters represented by the optimal pitch amplitude and phase shift that maximize the energy harvesting efficiency. The computational fluid dynamics (CFD) based on the $k-\omega-S S T$ turbulence model is implemented to find the optimal control parameters for a simultaneously heaving and pitching 2D wing. For the experimental investigation, a wind tunnel model is manufactured and used to perform the wind tunnel tests to prove the energy harvesting concept and validate the obtained CFD results. Since it wasn't feasible to generate sinusoidal vertical gust in the wind tunnel, the gust effect is modeled by a sinusoidal heaving motion of the wing. A robotic arm is used to perform the simultaneous heaving and pitching motions of the wing. The numerical results showed the significant effect of the control activation to increase the energy harvesting where an optimal efficiency of $67 \%$ is achieved at a gust amplitude of $0,5 \mathrm{~m} / \mathrm{s}$ and frequency of $0,4 \mathrm{~Hz}$. It was also found that an increase in the amplitude of the sinusoidal gust profile brings significant increment in the amount of energy harvested. Wind tunnel tests proved the concept of energy harvesting and exhibit the same trends of efficiency variation with pitch amplitude as that obtained through the numerical simulations. The obtained results showed that the energy harvesting flight technique is very promising regarding the improvement of the performance of mini-UAVs.
\end{abstract}

\section{Introduction}

A SMALL UNMANNED AERIAL VEHICLE usually flies in the lower levels of the atmospheric boundary layer. Various formations of rising air can form in that region due to the intricate interaction of natural obstacles and moving atmosphere as shown in Figure 1. It is well known that birds utilize those atmospheric motions for performance improvement in the form of extended endurance and range. Considering the fact that small unmanned aerial vehicles share the speed of flight and dimensions with natural flyers, bio-inspired flight techniques represent a great opportunity for significant energy savings of small unmanned aerial vehicles, as demonstrated recently by Gavrilovic. ${ }^{1}$ The concept of propulsive effect on a small flying vehicle while experiencing a vertical gust has been initially presented by Patel and $\mathrm{Kroo}^{2}$ and more recently by Gavrilovic et al. ${ }^{3,4}$ The concept states that a flying vehicle experiences a small propulsive force performing positive work on the aircraft due to the tilting of lift force which is again a consequence of vertical wind component. That propulsive force is acting in the opposite direction from drag and is directly responsible for the reduction in invested power. The effect in such atmospheric conditions can be even more amplified with instantaneous augmentation of lift force. The optimal effect can be achieved with an increase in the angle of attack with respect to drag generation. The work from Granlund et al. ${ }^{5}$ has shown that aerodynamic gust response

\footnotetext{
*Postdoctoral researcher, Department of Aerodynamics, Energetics and Propulsion, 10 Avenue Edouard Belin, 31400 Toulouse, France, AIAA Member.

${ }^{\dagger}$ Graduate student, Department of Aerodynamics, Energetics and Propulsion, 10 Avenue Edouard Belin, 31400 Toulouse, France.

${ }^{\ddagger}$ Associate Professor, Department of Aerodynamics, Energetics and Propulsion, 10 Avenue Edouard Belin, 31400 Toulouse, France.

$\S$ Full professor, Department of Aerodynamics, Energetics and Propulsion, 10 Avenue Edouard Belin, 31400 Toulouse, France, AIAA member.
} 
measurements can be conducted in steady freestream conditions by oscillating the test article. The apparent mass contribution due to the acceleration of flow is present in both streamwise oscillation and oscillating airfoil case. Therefore, this work will be mainly dealing with an oscillating foil, while some comparison with adequate gust conditions will be additionally verified concerning realistic turbulence spectrum. Moreover, the work will be performed with symmetric airfoil chord of $0.2 \mathrm{~m}$ and airspeed of $10 \mathrm{~m} / \mathrm{s}$, as a typical representative of a small unmanned aerial vehicle flight conditions. The present investigation on an oscillating airfoil is based on unsteady turbulent two-dimensional numerical simulations of the complex viscous flows with up to stall amplitude motions are considered. This study aims to determine the optimal aerodynamic parameters maximizing the propulsive efficiency achieved by a single airfoil at low Reynolds number of 150,000. In this paper, we first present a description of the aerodynamics of the oscillating airfoils with detailed characterization of different operating regimes: namely power extraction and propulsive regime as explained previously by Kinsey and Dumas. ${ }^{6}$ The inadequacy of a quasi-steady approach for the oscillating airfoil has been presented by Theodorsen ${ }^{7}$ in his classical work on unsteady aerodynamics. The present investigation confirms this inadequacy, extending the study to the strong non-linear behavior with the viscous flow and significant, although below stall, amplitudes of motion. Moreover, the study will be focused on non-zero lift flight conditions by assuming that aircraft flies with a certain mean constant lift coefficient. A mapping of propulsive efficiency in function of oscillating amplitude and frequency is then presented for a NACA 0015 airfoil and fixed Reynolds number that corresponds to a typical UAV flight. Furthermore, the impact of pitching amplitude, introduced as active control of a wing, is discussed, and its importance is emphasized through analysis of different flight scenarios. Accuracy of the numerical results presented here is thoroughly validated through a process that involves rigorous spatial and temporal convergence tests as well as comparison with experimental wind tunnel test campaign.

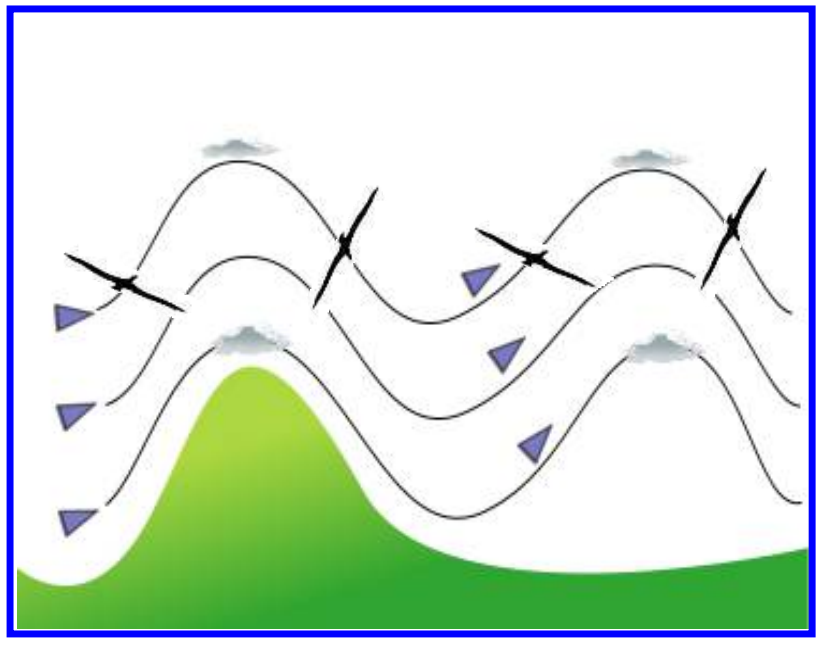

(a) Ridge lift

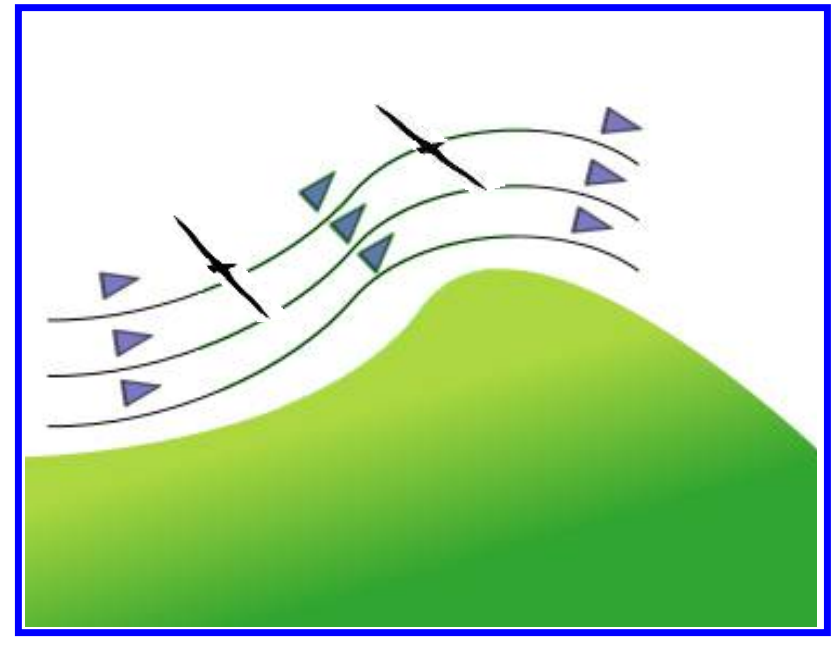

(b) Lee wave

Figure 1. Natural phenomena.

\section{Oscillating Airfoil Principle}

\section{A. Motion Description}

This work is dealing with an oscillating two-dimensional wing experiencing simultaneous rotation $\alpha_{c}(t)$ and vertical motion $y(t)$, as shown in Figure 2. The airfoil motion is expressed as where $k$ and $A_{y}$ are, respectively, the pitching and heaving amplitudes; $\Omega$ is the pitching velocity an; $\omega$ is the angular frequency $(2 \pi f$ where $f$ is in $\mathrm{Hz}) ; V_{y}$ is the heaving velocity; and the phase difference between two motions.

$$
\begin{gathered}
\alpha_{c}(t)=k_{\alpha} \sin (\omega t) \longrightarrow \Omega(t)=k_{\alpha} \omega \cos (\omega t) \\
y(t)=A_{y} \sin (\omega t+\phi) \longrightarrow V_{y}(t)=\dot{y}=A_{y} \omega \cos (\omega t+\phi) \text { where } A_{y} \omega=k_{g}
\end{gathered}
$$




\section{B. Operating Regimes}

An oscillating symmetric airfoil with $\alpha_{0} \neq 0$ as shown in Figure 3 can operate in two different regimes. This fundamental distinction is coming from the direction of the forces that are generated on the moving airfoil. Based on the imposed vertical motion and the upstream flow conditions, the airfoil experiences a heaving angle of attack $\alpha_{h}$ and an effective upstream velocity $V_{\text {eff }}$, as functions of time, expressed as follows:

$$
\begin{gathered}
\alpha_{h}(t)=\arctan \left(-V_{y}(t) / V_{\infty}\right) \\
V_{e f f}(t)=\sqrt{V_{\infty}^{2}+V_{y}^{2}(t)}
\end{gathered}
$$

The total angle is a sum of an imposed initial angle, input rotation and angle induced by vertical motion as follows:

$$
\alpha_{T}=\alpha_{0}+\alpha_{c}+\alpha_{h}
$$

The maximum value of heaving angle of attack is expected to have a major impact on peak forces generated. The maximum total angle of attack is approximated by the modulus of its quarter period value (for $\phi=90^{\circ}$ ). In order to qualify the effect of imposed motion on the flow regime, we define a feathering parameter, in a similar way as in work of Kinsey and Dumas ${ }^{6}$ and Anderson et al. ${ }^{8}$

$$
\chi=\frac{k_{\alpha}+\alpha_{0}}{\arctan \left(A_{y} \omega / V_{\infty}\right)}
$$

Based on the assumption that the unsteady effects are not present, one can show that $\chi>-1$ is associated with propulsion effect on the airfoil with $\alpha_{T}(T / 4)>0$. On the contrary, the opposite case whereas $\chi<-1$ corresponds to power extraction regime. As an example of the first case is shown in the schematic representation of Figure 3, which represents a time sequence viewed in a reference frame moving with the far-field flow at $V_{\infty}$, so that the total angle of attack is shown visible from the apparent trajectory of the airfoil. The figure shows that the resultant aerodynamic force is having horizontal component acting with positive $x$-direction (effective thrust noted in figure as $\Delta L$ ).

\section{Propulsive Efficiency of an Oscillating Airfoil}

The instantaneous harvested power when $\chi>-1$ is coming from the propulsive force $\Delta L$ which performs a positive work on the airfoil. We further define the propulsive efficiency $\eta$ as the ratio of mean net forces for one period in the $x$-direction between the case of coupled pitching and heaving airfoil and the case with no control input (i. e. no pitch) with only heaving. The comparison between cases with different control inputs and case with only heaving (or vertical wind effect) will be performed with the same frequency for both motions and the same heaving amplitude.

$$
\eta=1-\frac{\overline{C_{x, i}}}{\overline{C_{x, N}}}=1-\int_{0}^{1} \frac{C_{x, i}(t)}{C_{x, N}} d(t / T)
$$

The efficiency defined in this way describes the amount of mean drag reduction with introduction of pitching control. Basically, when analyzed through flight parameters, the efficiency is equivalent to a percentage of reduced invested power when compared to altitude hold flight.

\section{Numerical Analysis}

In this study, we rely on a computationally intensive method, namely on an unsteady Navier-Stokes solver. The reason behind that is a need for modeling of the unsteady flows around moving bodies with viscid effects. An initial attempt to model the aerodynamics of a moving body could be to use the instantaneous force coefficients from stationary data matching the instantaneous angle of attack. However such a quasi-steady approach would lack accurate results even for smooth cases far away from stall. It will be later shown that unsteady effects are significant for both lift and drag evolution and that they grow with frequency increase, as expected. 


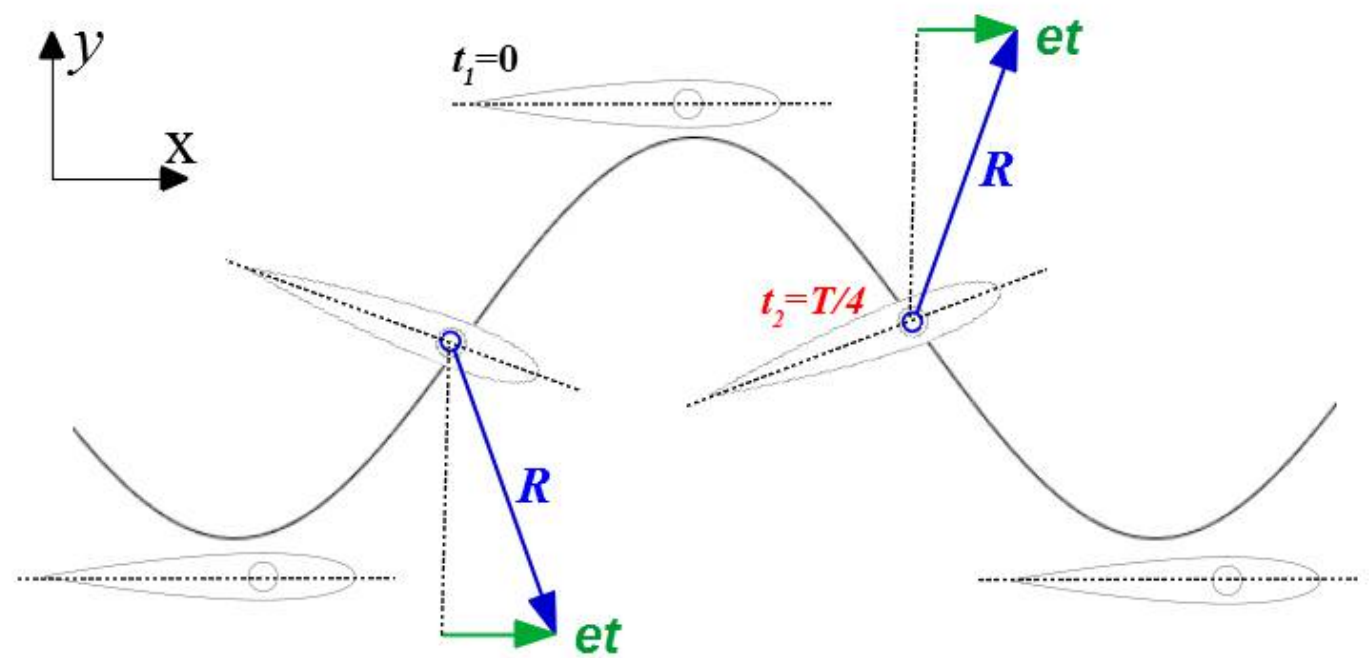

Figure 2. Propulsive regime of an oscillating airfoil for $\chi>-1$ (et=effective thrust).

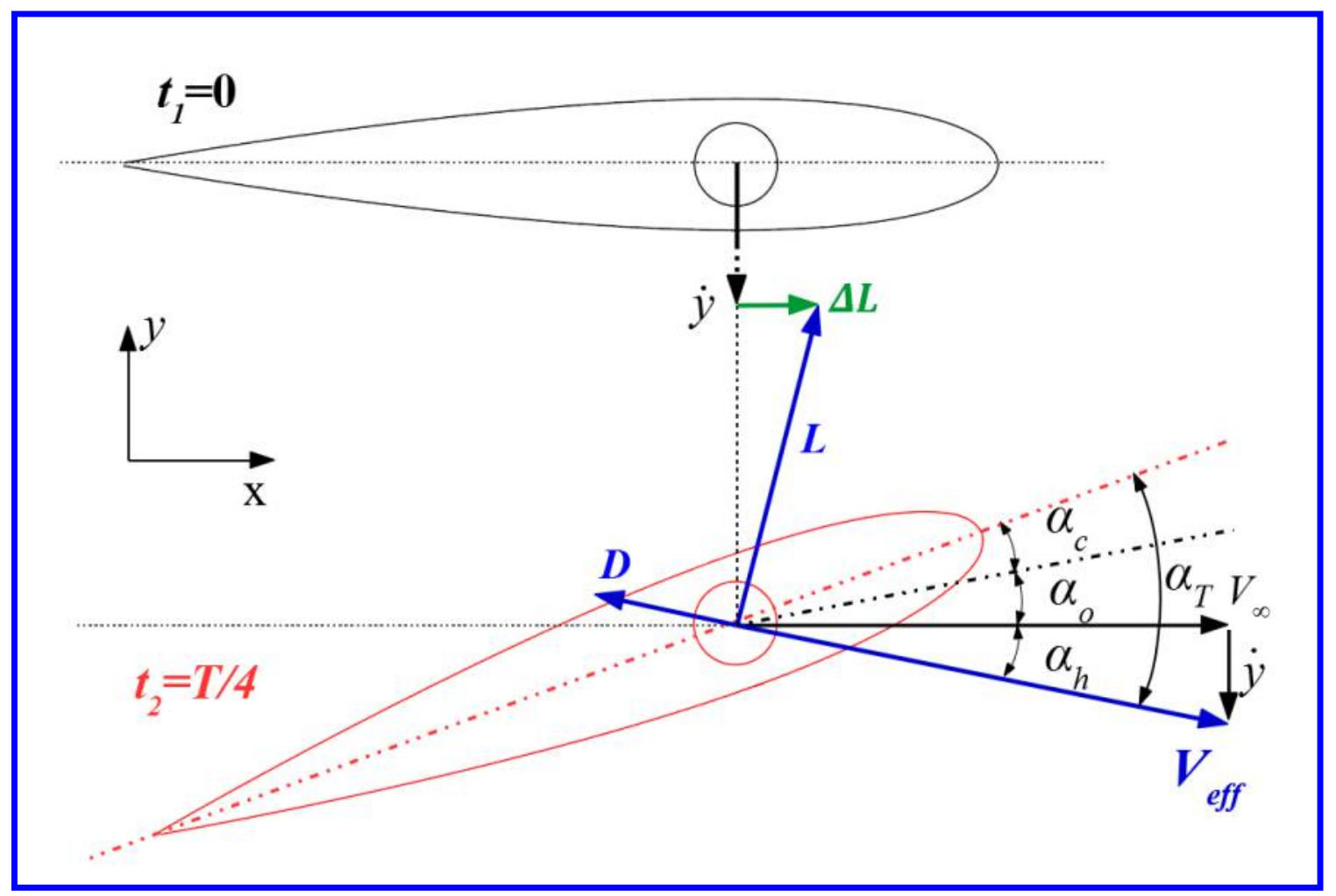

Figure 3. Propulsive regime for $\alpha_{\max }=\alpha(T / 4)$.

\section{A. Flow Solver}

The case under consideration is unsteady two-dimensional turbulent flow. The commercially available CFD solver FLUENT 6.1 is implemented using a $k-\omega-S S T$ turbulent model to investigate the complex aerodynamic characteristics of the flow around the heaving and pitching airfoil. The SIMPLE scheme is selected for the pressure velocity coupling using the pressure-based segregated solver. Least square cell-based method is used 
to compute the gradients. Second-order upwind scheme for the convection term and second-order implicit time-stepping are used. The heaving and pitching motions are modeled using the mesh motion technique available in fluent software. The heaving motion is implemented through the translational motion of the outer domain while for the pitching motion; a sliding mesh interface is used to allow for the rotational motion of the inner circular domain with respect to the outer translating domain. The prescribed translational and rotational motions are defined to the solver via a User Defined Function (UDF).

\section{B. Mesh Strategy}

The current mesh is generated based on the previous work of Kinsey and Dumas. ${ }^{6}$ The domain is divided into two cell zones by a circular non-conformal sliding mesh interface as shown in Figure 4. The inner circular domain around the airfoil is used to impose the control pitching motion through the rotation of this domain using the sliding mesh technique. Special care is taken to achieve a smooth transition between the elements at the interface. Figure 4 also shows a schematic of the computational domain dimensions and the generated grid respectively. The generated structured grid has a minimum element quality of 0.9 to ensure solution accuracy. The first layer height over the airfoil surface is implemented to achieve a $y^{+} \approx 1$ and full resolution of the boundary layer.

\section{Validation}

To validate our numerical predictions, rigorous self-consistency tests were carried out to assure satisfactory independency of the force predictions with respect to both mesh and time discretization. A grid and time independence studies are performed to select the optimum mesh size and time step for the best accuracy and minimum computational time. Both studies are conducted under flow condition with Reynold number of 140,000, reduced frequency 0.05 , non-dimensional heaving amplitude of 0.05 and pitching amplitude of $7^{\circ}$. The simulation time is taken to be $10 \mathrm{~T}$ in order to reach the long-term periodic flow response after the impulsive start in each simulation. The percentage change in the mean and peak force coefficients with both mesh size and the time step is shown in Table 1. Three different mesh resolutions were used: a coarse mesh with 28,000 elements, a medium mesh with 72,000 elements and a fine mesh with 166,000 elements. To establish time-discretization independency, three levels of time steps per cycle were also considered: 100, 500 and 1000. It can be seen from Table 1 that for a medium mesh, 500 time steps per cycle yields time accurate predictions for both mean and peak values. Furthermore, results show that our medium-mesh resolution provides satisfactory accuracy in space, as far as force predictions concerned. Indeed, for our three test cases, differences between medium- and fine-mesh results are small, with variation less than $1 \%$ on both peak and mean force coefficients.

\begin{tabular}{cccccc}
\hline \hline & Time steps/cycle & No. of Elements & \% change $\overline{C_{d}}$ & \% change $\widehat{C}_{d}$ & \% change $\widehat{C_{l}}$ \\
\hline \multirow{3}{*}{ Mesh Study } & 500 & 28000 & - & - & - \\
& 500 & 72000 & 3.8 & 3.6 & 1 \\
& 500 & 166000 & 0.06 & 0.8 & 0.6 \\
\hline \multirow{3}{*}{ Time step study } & 100 & 72000 & - & - & - \\
& 500 & 72000 & 1.3 & 6 & 4.3 \\
& 1000 & 72000 & 0.75 & 0.4 & 0.7 \\
\hline \hline
\end{tabular}

Table 1. Validation through space and time refinement for an airfoil in propulsive regime.

The following example demonstrates the application of the Richardson extrapolation in conducting a grid convergence study. The objective of the CFD analysis was to determine the mean drag and lift coefficients for an oscillating airfoil. The flow field is computed on three grids, each with 2.5 times the number of grid elements. Each solution was properly converged with respect to iterations. Figure 5 shows the plot of drag coefficient with varying grid spacing. As the grid spacing reduces, the drag coefficient approaches an asymptotic zero-grid spacing value. Based on this study we could say that the drag coefficient for the oscillating airfoil is estimated to be $\overline{C_{d}}=0.02594$ with an error band of $0.01 \%$. Once again, the very good 


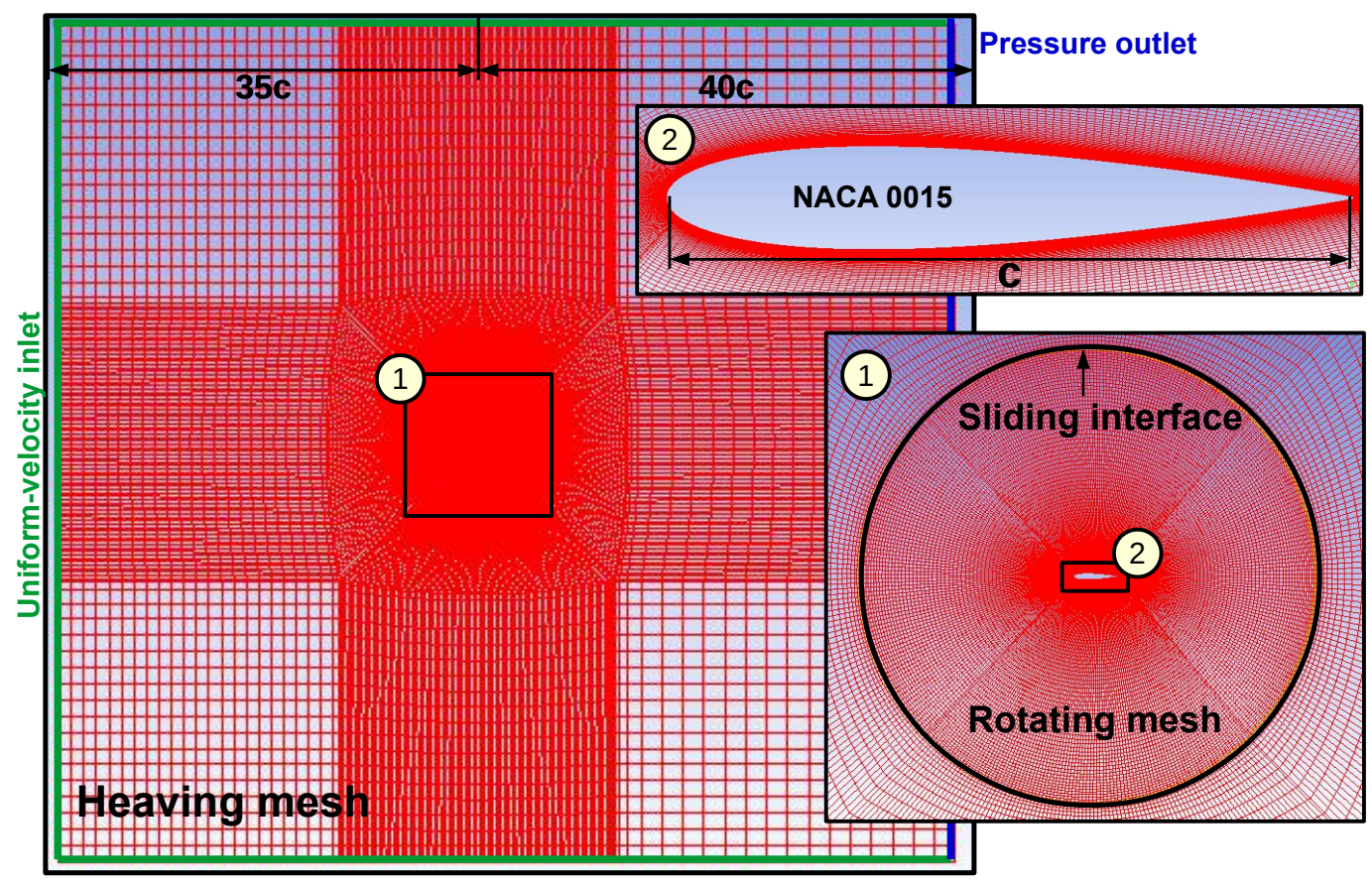

Figure 4. Grid details with two zoom levels showing its circular sliding interface.

agreement between medium- and fine-discretization results led us to adopt 72,000-cell mesh and 500 time steps per cycle.

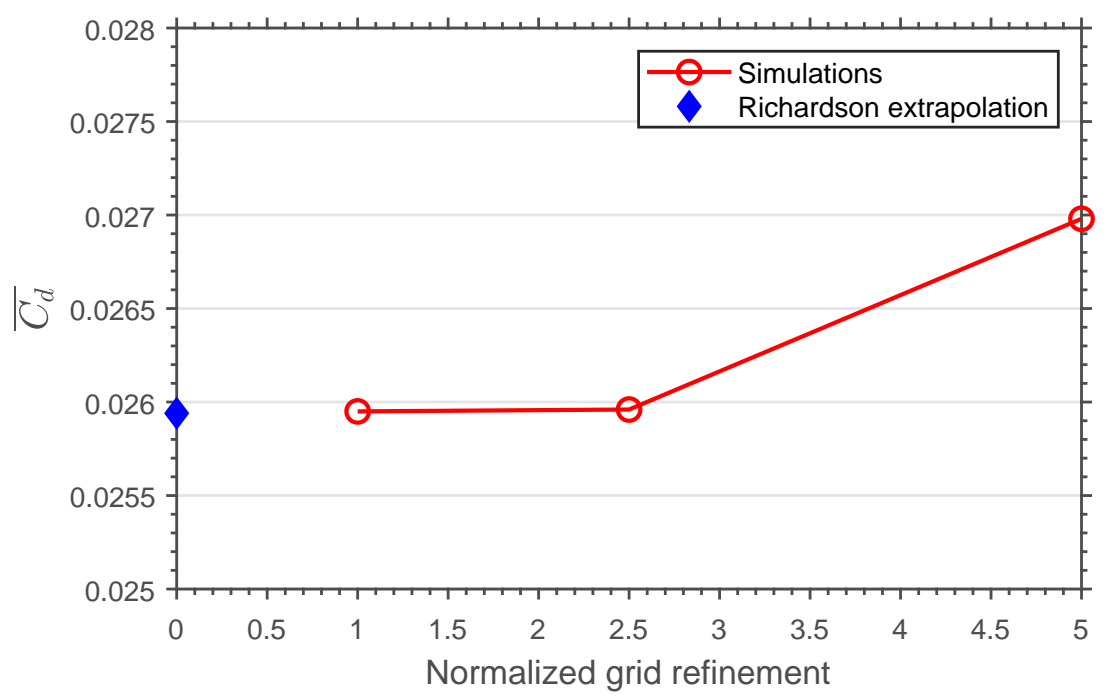

Figure 5. Richardson extrapolation for grid refinement study. 


\section{Results of the Propulsive Performance}

\section{A. Flight Cases}

Results related to four different flight cases are summarized in Table 2. It should be noted that all the cases in this analysis are performed with an initial angle of attack of three degrees, in the purpose of maintaining a constant mean, non-zero lift coefficient. The first representative case is a mean level of drag required for flight performed in steady conditions. This flight will be used as a reference to all other flights analyzed. The second flight considers a flight with fixed-stick within vertical wind profile with amplitude shown in Table 2. It illustrates the potential benefits in the case of no control. It can be concluded that a significant benefit would also be present with just flying through the sinusoidal environment without any control input. The next flight assumes usage of the regulator for maintaining constant lift coefficient. This flight consumes 5.4 $\%$ more power when compared to reference flight. It reflects on the auto-stabilization mode of autopilot in loitering at a constant altitude. Moreover, we recall a very general expression for lift coefficient shown in Eq. 8 , which can be found in work of Bonnet. ${ }^{9}$ In order to maintain constant lift coefficient, it can be seen from Eq. 8 that for any predefined motion of pitching or heaving one can find a solution for the other motion where the lift part of the added mass term and vortex emission term neglect each other. The solution for pitching motion has been presented in Eq. 9. It consists of two parts, where the first represents a motion which neglects steady lift from heaving while the second part eliminates unsteady effects. The stabilization control in pitching for any test case with heaving has been shown in Eq. 9 and it has been obtained from a general equation for lift coefficient with pitching and heaving (see Eq. 8). The effectiveness of control has been shown in Figure 6. The figure shows the evolution of lift and drag coefficient in function of parameter $a$ (from Eq. 9) which has a task do eliminate unsteady effects.

$$
\begin{gathered}
C_{l}=C_{l 0}+\overbrace{\frac{\pi}{2}\left(\dot{\alpha}-\ddot{A}_{y}+\left(\frac{1}{2}-x_{g}\right) \ddot{\alpha}\right)}^{C_{l} \text { added mass term }}+\overbrace{2 \pi\left(\alpha-\alpha_{0}+\dot{\alpha}\left(\frac{3}{4}-x_{g}\right)-\dot{A_{y}}\right) C}^{C_{l} \text { vortex emission }} \\
\alpha_{c}(t)=k_{\alpha} \sin (\omega t+\phi)+k_{\alpha} a \cos (\omega t+\phi)+\alpha_{0}
\end{gathered}
$$

\begin{tabular}{ccc}
\hline \hline Flight conditions & $\overline{C_{x}}$ & $\%$ change in $\overline{C_{x}}$ wrt steady flight condition \\
\hline Steady flight $k_{g}^{*}=0$ & 0.0167 & - \\
Stick-fixed $k_{g}^{*}=0.05$ & 0.01205 & $-27.8 \%$ \\
Const. $C_{l}, k_{g}^{*}=0.05$ and $k_{\alpha}=-2.85^{\circ}$ & 0.0176 & $5.4 \%$ \\
Energy-harvesting $k_{g}^{*}=0.05$ & 0.0064 & $-61.7 \%$ \\
\hline \hline
\end{tabular}

Table 2. Flight cases with $\alpha_{0}=3^{\circ}$.

Finally, the fourth flight brought a saving of around $60 \%$ when compared to a reference value. This case demonstrates the usage of optimal control in pitching while performing heaving motion. The mission of pitching motion is to optimally increase the effective thrust with respect to drag increase.

\section{B. Frequency Effect}

The purpose of this analysis is to reveal the effect of frequency change on the overall efficiency of the cycle. Once again all the simulations in this paragraph are performed with a constant initial angle of attack of three degrees. Each study case of this analysis was performed with a constant non-dimensional velocity of heaving. For a single frequency study, the amplitude of pitching motion was varied with an objective to find an optimal solution. The overall trend in Figure 7 shows that the highest achieved efficiency in function of optimal pitching grows significantly with drop-in frequency. The reason behind efficiency increase is significant drop in mean net drag coefficient with decreasing frequency. It can also be seen that optimal pitching amplitude reduces with increasing frequency.

The reason behind significant efficiency drop can be found in Figure 8 which illustrates the evolution of lift and effective thrust coefficients during one cycle. It can be seen that for fixed heaving amplitude 


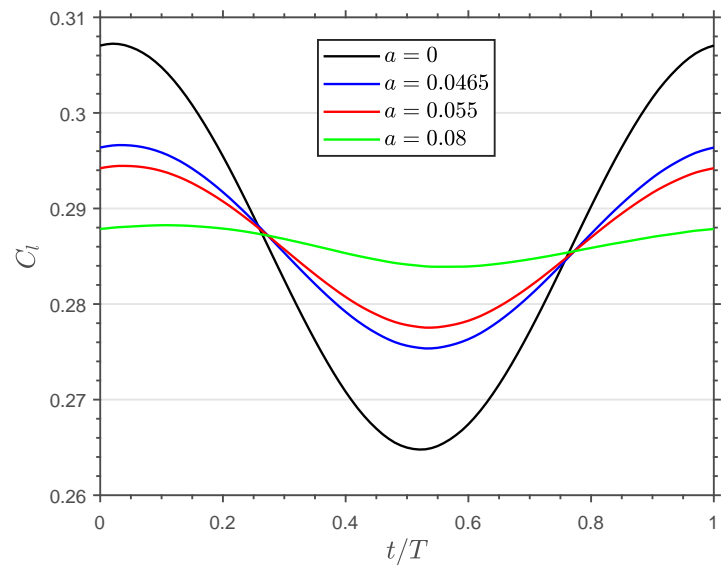

(a) Efficiency in function of pitch input

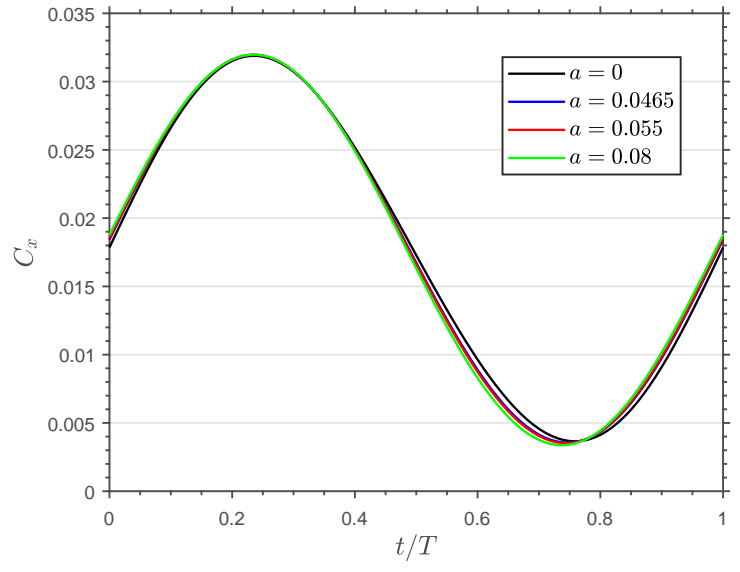

(b) Drag coefficient for one period

Figure 6. Constant lift coefficient with $k_{g}^{*}=0.05, k_{\alpha}=2.85^{\circ}$ and $k=0.05$.

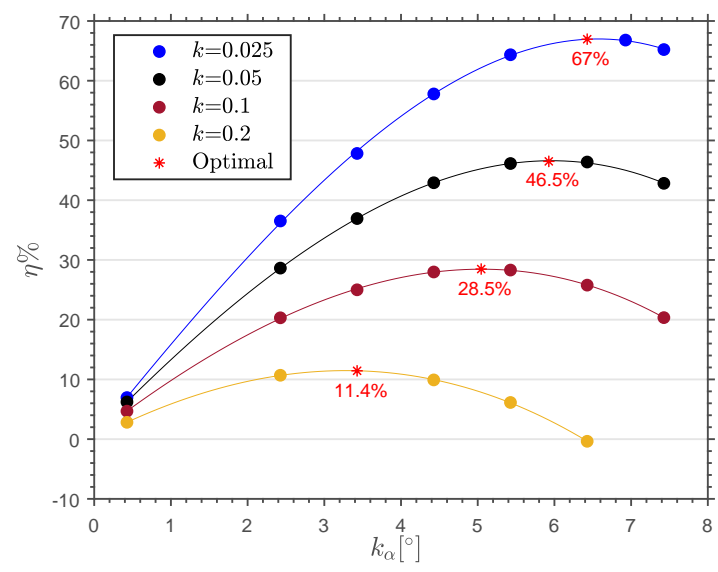

(a) Efficiency versus pitch input

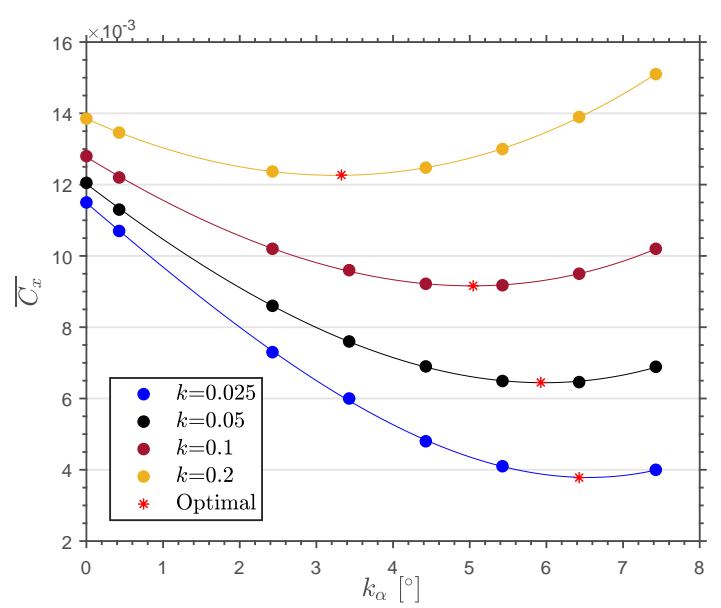

(b) Mean drag coefficient

Figure 7. Frequency effect with $\alpha_{0}=3^{\circ}, V_{\infty}=10 \mathrm{~m} / \mathrm{s}, k_{g}^{*}=0.05$ and $\phi=90^{\circ}$.

the airfoil achieves significantly greater peaks in the lift as the frequency drops. Consequently, the effective thrust coefficient is increased during the cycle. The asymmetry in the effective thrust coefficient between the first and the second half of the period is due to the existing initial (cruise) angle of attack.

\section{Amplitude Effect}

The purpose of this subchapter is to reveal the effect of oscillating magnitude on the overall efficiency of the cycle. All simulations in this study have been performed with an initial angle of attack of $3^{\circ}$ and nondimensional heaving amplitude of 0.1. Again, like in the previous analysis, for a single heaving amplitude study, the amplitude of pitching motion was varied to find an optimal solution. The overall trend in Figure 9 shows that the highest achieved efficiency in function of optimal pitching grows significantly with raise in amplitude of heaving.

The reason behind efficiency increase is a significant drop in mean net drag coefficient with increasing amplitude of heaving. Figure 10 shows that airfoil achieves higher lift coefficient and, therefore, higher effective thrust coefficient with higher heaving magnitude. The reason behind this is that the induced angle of attack from heaving is strongly affected by the amplitude of heaving. In the purpose of summarizing results, this chapter also introduced Figure 11 which now describes the overall trend in optimal efficiency in 


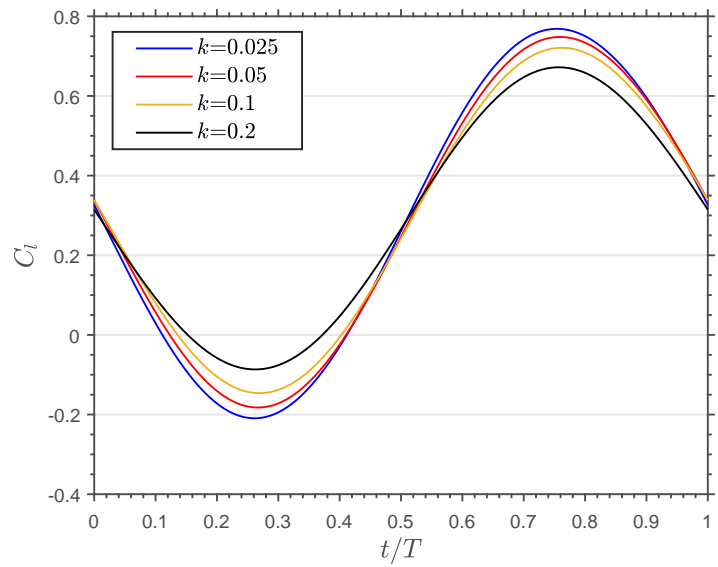

(a) Lift coefficient

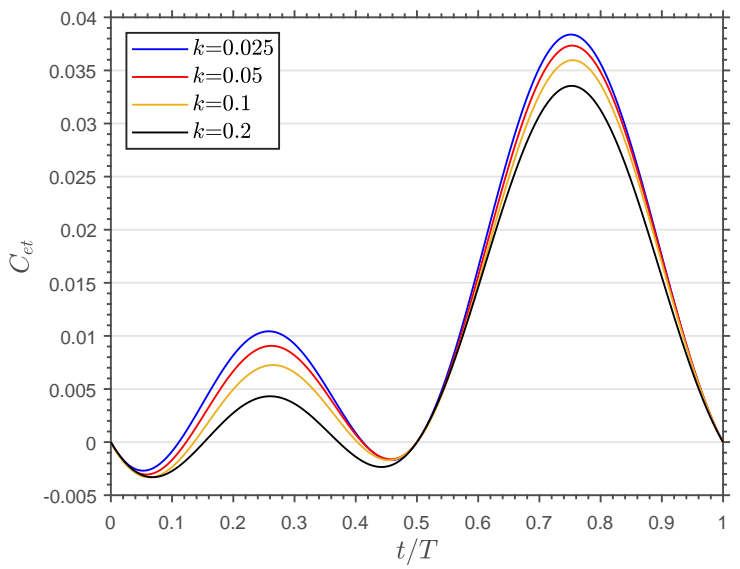

(b) Effective thrust coefficient

Figure 8. Lift and effective thrust coefficient for the frequency effect analysis. $k_{g}^{*}=0.05$ and $k_{\alpha}=2.4^{\circ}$.

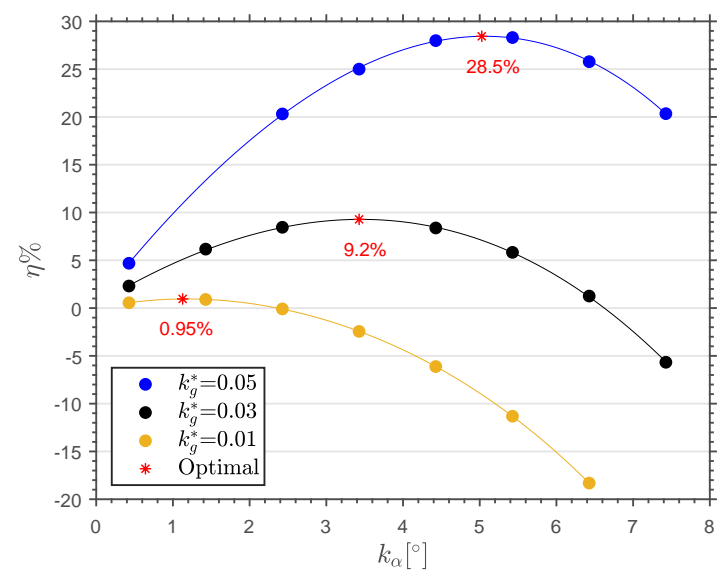

(a) Efficiency versus pitch input

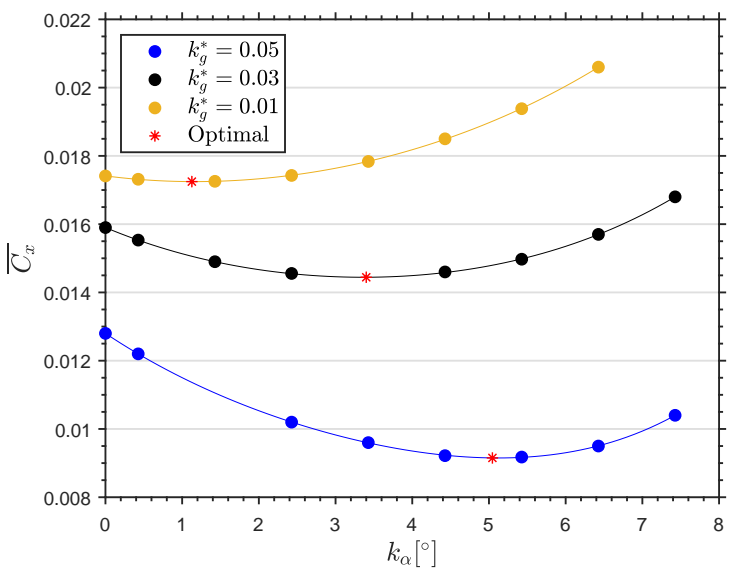

(b) Mean drag coefficient

Figure 9. Amplitude effect on efficiency with $\alpha_{0}=3^{\circ}, V_{\infty}=10 \mathrm{~m} / \mathrm{s}, k=0.1$ and $\phi=90^{\circ}$.

function of both amplitude and heaving. Totally three magnitudes of heaving with several frequencies are presented and will be denoted in the future text as small, medium and high. It can be seen in the figure that small amplitude of heaving provided a relatively low level of optimal efficiency, starting with $4 \%$ for the highest frequency and reducing towards less than $1 \%$ for the highest frequency. On the other side, medium and high amplitudes have provided a significantly higher level of optimal efficiency while still keeping the decreasing trend with increasing frequency. It is important to notice that for the chosen conditions in this study all the non-dimensional magnitudes of heaving motion bellow 0.01 can be considered to have negligible effect on optimal efficiency. In this study those conditions correspond to a vertical wind component less than $0.1 \mathrm{~m} / \mathrm{s}$.

To gain some insight into the propulsive effect of an oscillating airfoil, sinusoidal cycles with two different frequencies are presented in Figure 12 and Figure 13. It can be seen that both representations have the same induced angle of attack from heaving and the same total angle of attack evolution, as the reduced amplitude of heaving is equal. The only difference in this comparison is in a reduced frequency which changes from 0.025 up to 0.1. The color bar on the figure is present to illustrate the advancement of a cycle according to time within one period. It can be seen on the graphs that the unsteady effects are considerably stronger for the case with higher frequency as the drag envelope is significantly wider. The cycle also shows a region where drag coefficient changes its sign into negative, thus having a horizontal force which now acts in positive 


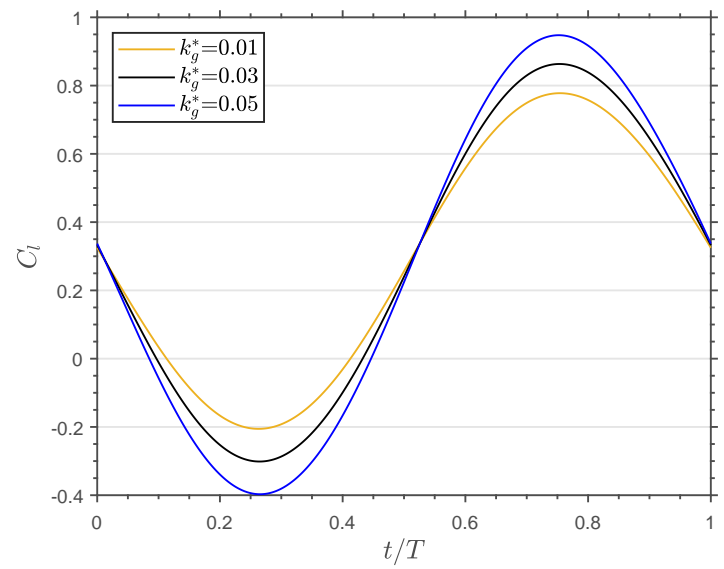

(a) Lift coefficient for one period

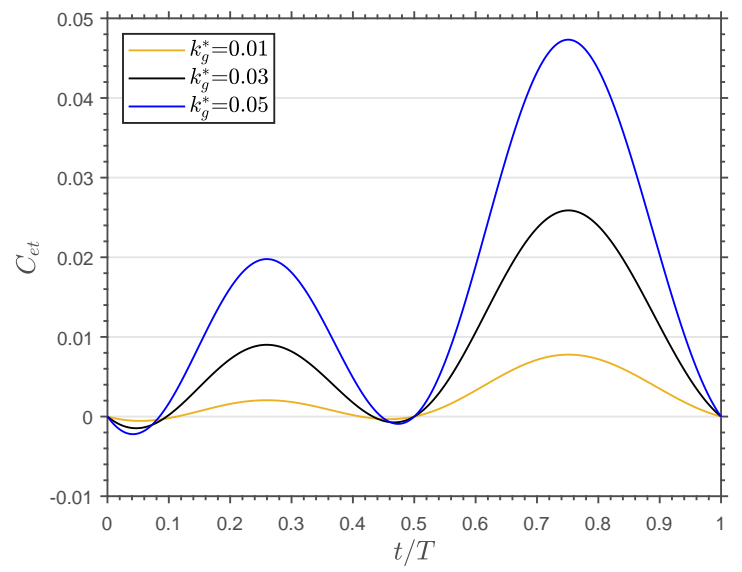

(b) Effective thrust coefficient for one period

Figure 10. Amplitude effect on lift and effective thrust with $\alpha_{0}=3^{\circ}, V_{\infty}=10 \mathrm{~m} / \mathrm{s}, k=0.1$ and $\phi=90^{\circ}$.

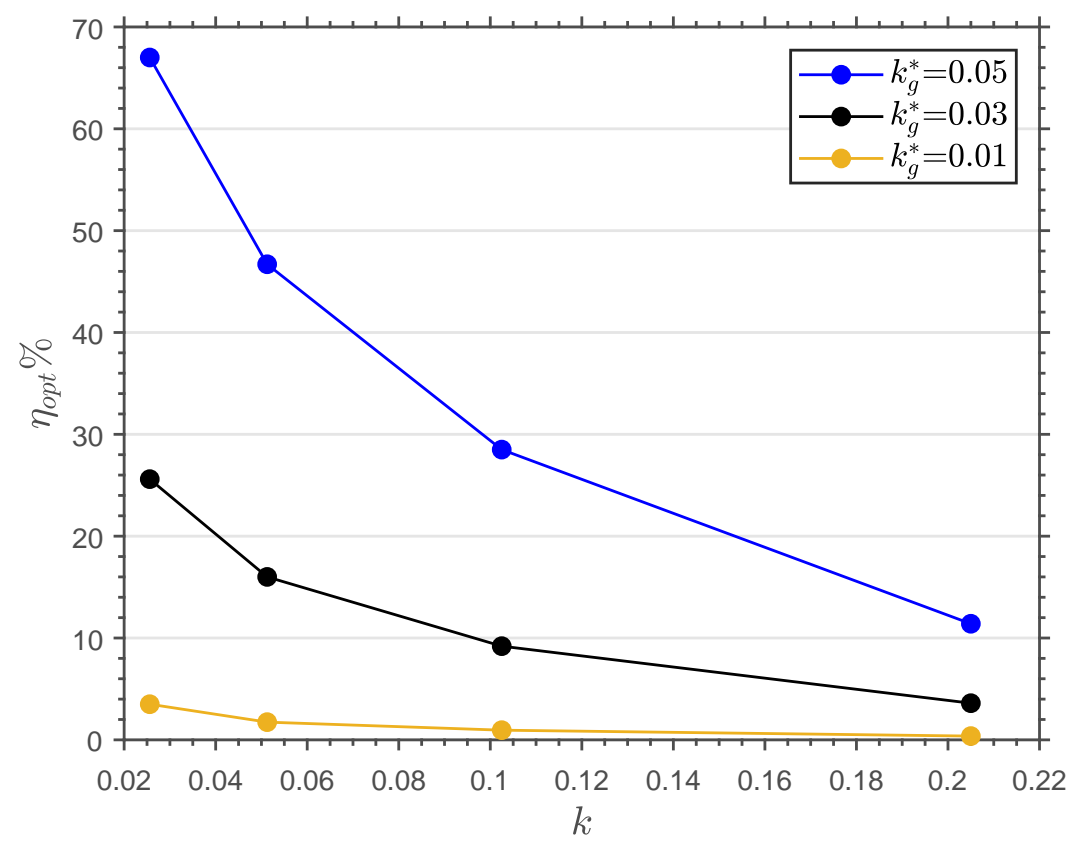

Figure 11. Optimal efficiency in function of heaving speed and frequency.

(forward) direction. In a real flight that would mean that the aircraft has an excessive amount of thrust. It is also interesting to notice that the overall level of the drag curve for low frequency is on the lower mean level when compared to a higher frequency. This conclusion is coming from the fact that the added mass effects are stronger with high-frequency oscillations. Therefore, the overall efficiency of a low-frequency cycle is higher when compared to high-frequency cycle.

\section{Shift Phase Effect}

Figure 14 shows dependence of the propulsive efficiency $\eta$ upon $\phi$, the phase lag between the heaving and the pitching modes. Best performance is obtained in the vicinity of $\phi=90^{\circ}$. This finding has been also previously found in the work by Zhu. ${ }^{10}$ The first analysis has been performed for the lower magnitude 


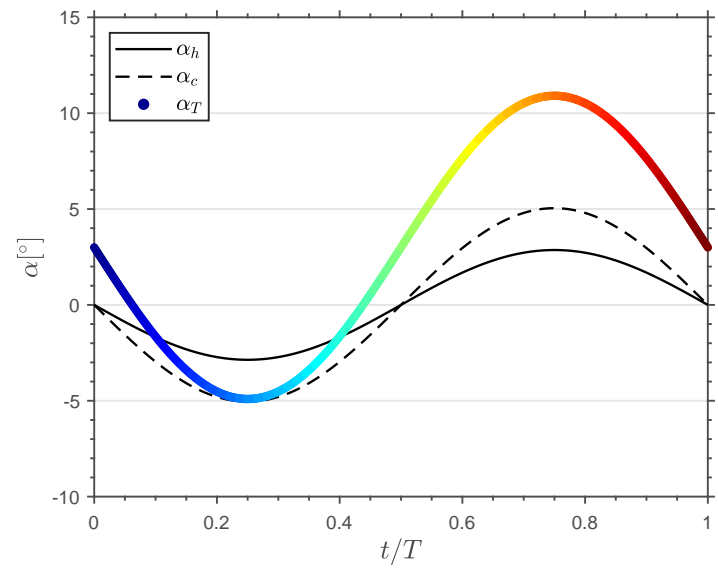

(a) Angles

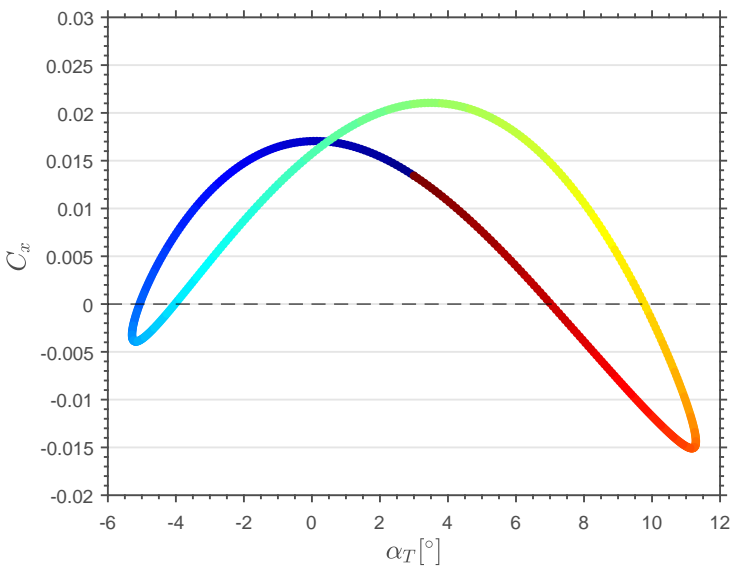

(b) Drag coefficient evolution

Figure 12. Force coefficient in $x$-direction for $\alpha_{0}=3^{\circ}, k_{g}^{*}=0.05, k=0.025(f=0.4 H z)$ and $k_{\alpha}=k_{\alpha(o p t)}=5.4^{\circ}$.

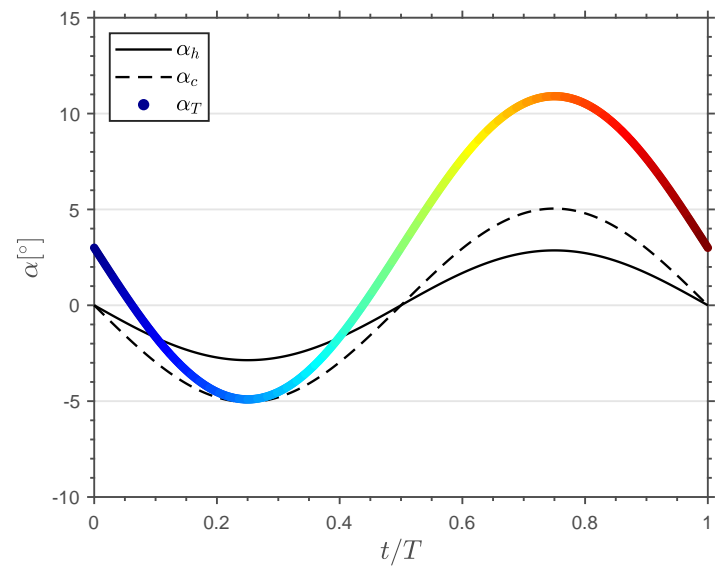

(a) Angles

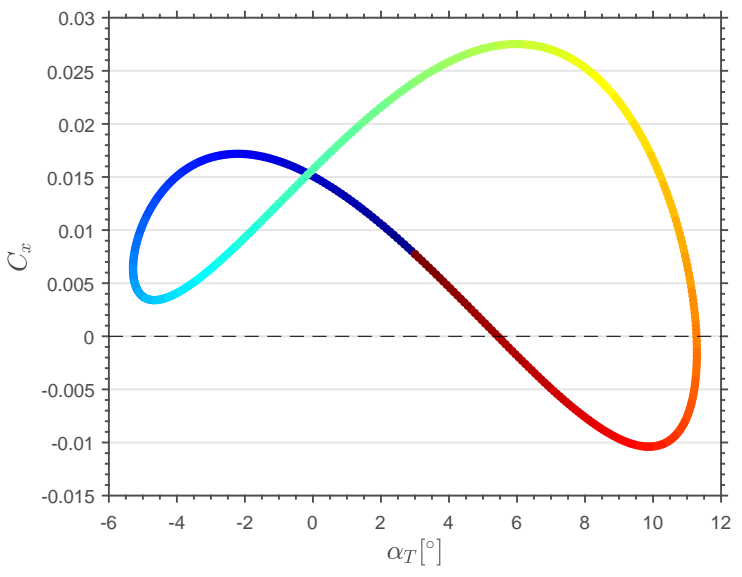

(b) Drag coefficient evolution

Figure 13. Force coefficient in $x$-direction for $\alpha_{0}=3^{\circ}, k_{g}^{*}=0.05, k=0.1(f=1.6 H z)$ and $k_{\alpha}=k_{\alpha(o p t)}=5.4^{\circ}$.

of heaving where low efficiency is to be expected. On the other side, the other case took into account significantly higher magnitude of heaving with maximum efficiency as high as $30 \%$. Both study cases have been performed with a constant mean lift coefficient and have confirmed that the optimal shift in phase is $90^{\circ}$ and not depending on the amplitude or frequency of oscillations.

\section{E. Heaving versus Wind}

The analysis presented in this paragraph is related to the comparison of two cases, of which, the first related to an airfoil experiencing a wind profile, and the second, an airfoil with a motion that provokes the same induced angle of attack like the one from the wind profile. The study is trying to quantify the difference in mean drag level between the cases, especially for the turbulence levels that can be experience in real UAV flight. The spectrum used in this study has been developed by Kaimal ${ }^{11}$ from the long-term experimental study over the flat homogeneous terrain. Although the Kaimal spectrum is empirical, it incorporates several factors such as terrain roughness, wind shear and mean wind magnitude. The spectrum generated in this work has been constructed with turbulence intensity of $30 \%$, which is very unlikely to be experienced in real life.

The results represented in Figure 15 are two blue points with star markers presented in Figure 16. Those 


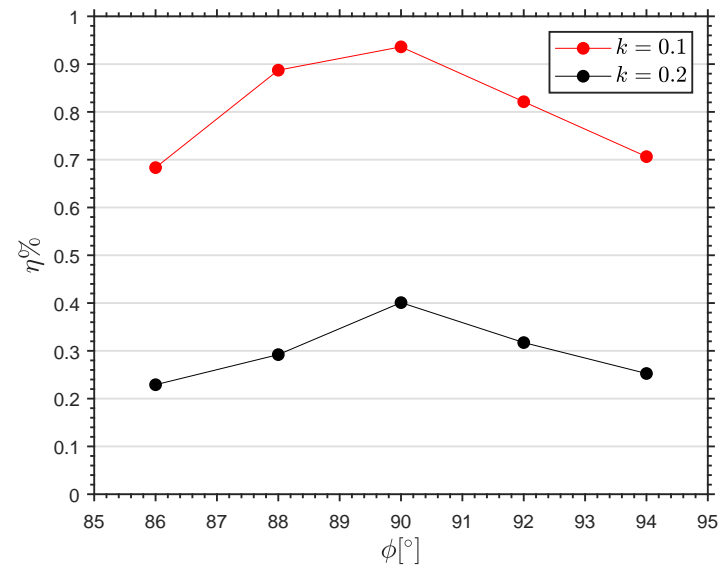

(a) $k_{g}^{*}=0.01$ and $k_{\alpha}=k_{\alpha(o p t)}$

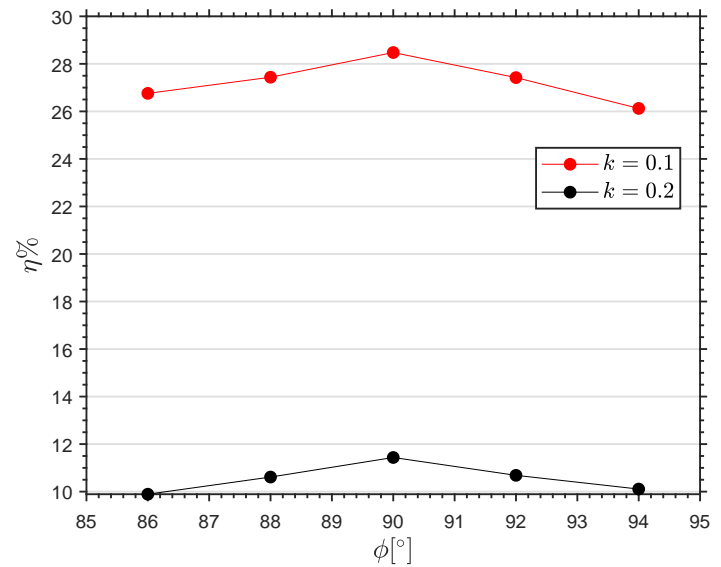

(b) $k_{g}^{*}=0.05$ and $k_{\alpha}=k_{\alpha(o p t)}$

Figure 14. Efficiency with constant mean lift coefficient in function of shift phase.

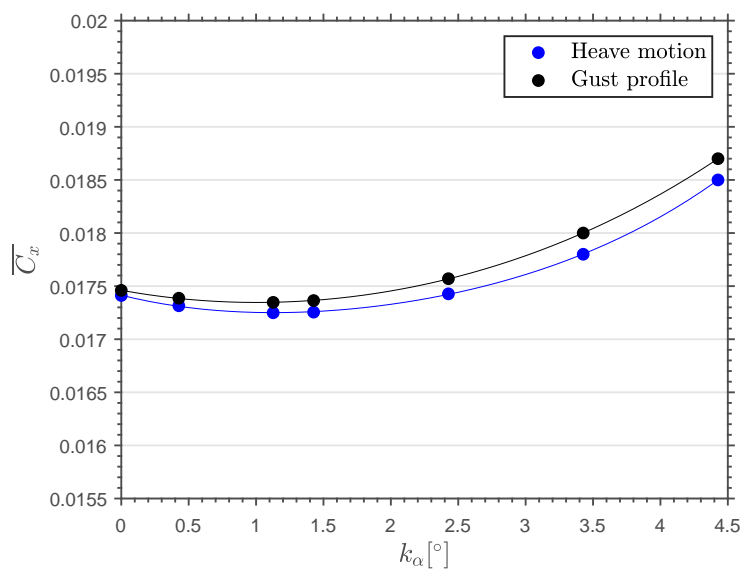

(a) $k_{g}^{*}=0.05, k=0.0065$

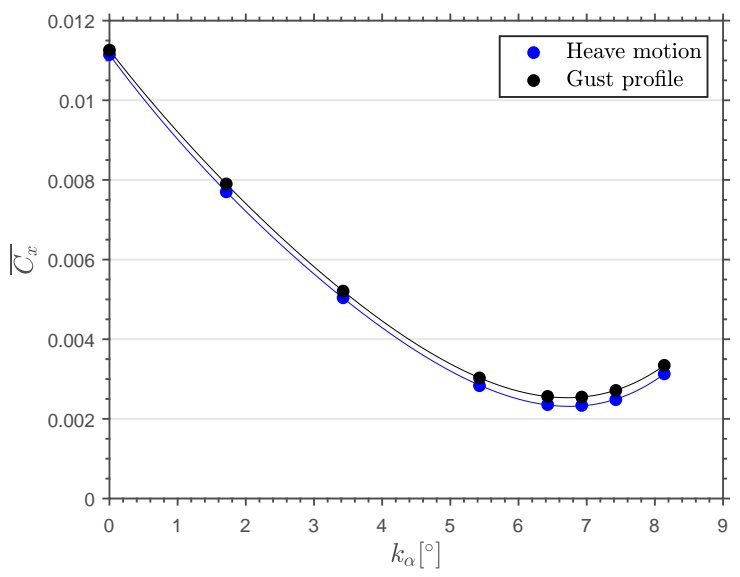

(b) $k_{g}^{*}=0.01, k=0.1$

Figure 15. Heaving motion versus wind profile.

two points are chosen as they belong to the overall shape of the turbulence spectrum for turbulence intensity of $30 \%$. The first point can be characterized as the low-frequency high amplitude, while the second one as high-frequency low amplitude. The results presented in Figure 16 show that the difference in mean drag coefficient for two previously chosen points from the spectrum and variation of control input is less than $5 \%$ in both cases. This conclusion is in agreement with results both performed theoretically and experimentally by Granlund et al., ${ }^{5}$ by claiming that aerodynamic gust response measurements can be conducted in steady freestream facilities by oscillating the test article. The third point used in this test and marked with red star in Figure 16 is characterized as high-frequency high-amplitude representing the scenario unlikely to be encountered in real life. The comparison between cases of gust profile and heaving motion with the equal induced angle of attack showed considerable difference in a mean drag coefficient of around 20\%. Despite the difference in drag level, the curve trends did not differ significantly by pointing out towards similar optimum control point. The analysis concludes that the aerodynamic effects of an airfoil penetrating a gust can certainly be represented by an oscillating airfoil in a freestream, even for frequencies and magnitudes that are possible but unlike to be experienced in real life. 


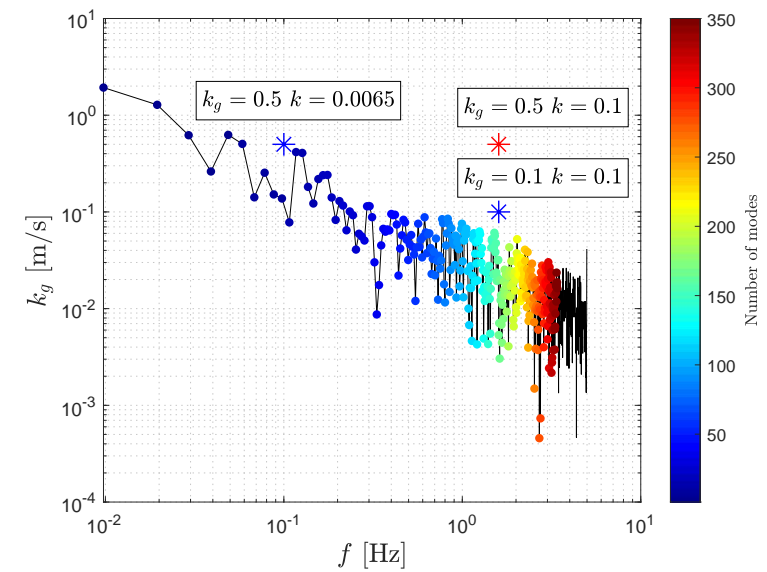

(a) Kaimal wind profile spectrum for $T_{i}=30 \%$

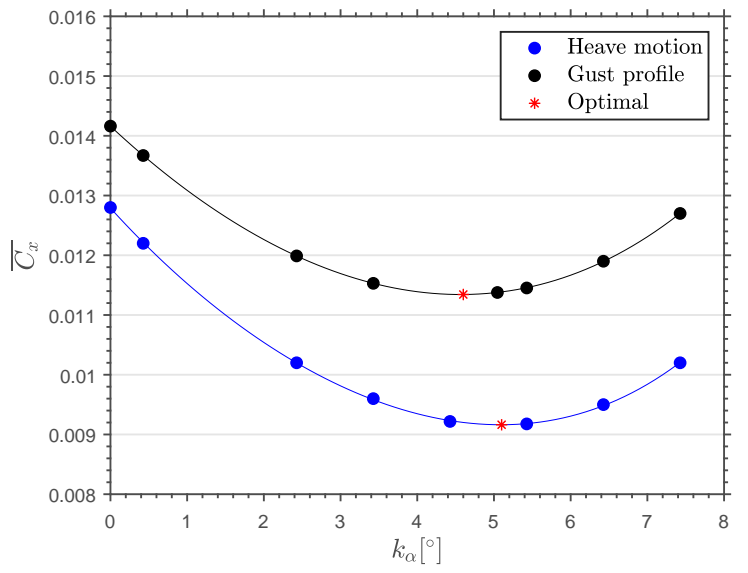

(b) $k_{g}^{*}=0.05$ and $k=0.1$

Figure 16. Wind profile versus heave motion.

\section{Experimental Verification}

Wind tunnel tests for heaving and pitching wing are performed at the wind tunnel facility available at the department of aerodynamics, energetics, and propulsion at ISAE-SUPAERO. The wind tunnel used is a closed-circuit tunnel with a maximum speed of $25 \mathrm{~m} / \mathrm{s}$ with the test cross section dimensions $80 \times 120 \mathrm{~cm}$ as shown in Figure 17. The motivation of the experimental investigation was to prove the concept of energy harvesting and to validate the obtained CFD results. In real life situation, the UAV should be performing pitching motion while experiencing sinusoidal vertical gust. But, for wind tunnel testing, it was not feasible to generate sinusoidal vertical gust, thus the vertical gust effect is modeled by heaving motion of the airfoil. The heaving and pitching motions of the wing are performed by using a robotic arm attached to the airfoil pitching center.

\section{A. Experimental Setup}

As a first step, a wind tunnel model of NACA 0015 wing shown in Figure 21 is fabricated having a span of $80 \mathrm{~cm}$ and a chord length of $20 \mathrm{~cm}$ as used in the CFD simulations. Initially, the wing shape has been cut from expanded polystyrene foam to minimize its weight, while afterward the wing surface is covered with a polymerized carbon fiber layer providing a smooth surface. A hole is made along wingspan at the pitching center position, where a spar is placed, passing through the whole wing towards the connection with the robotic arm as shown in Figure 21. A new test section wall shown in Figure 21 is manufactured to allow for mounting the wing in the test section and performing the required heaving and pitching motions. The new test section wall dimensions are $120 \times 120 \mathrm{~cm}$ and it has a horizontal slot which is $30 \mathrm{~cm}$ in length and 5 $\mathrm{cm}$ in width. The test section wall facilitates mounting of the wing inside the test section, where the wing is connected to the robot and positioned vertically while passing through the horizontal slot in the test section wall. Moreover, the horizontal slot allows for the heaving motion of the wing inside the test section.

The motivation of using a robotic arm to perform simultaneous sinusoidal heaving and pitching motion, arises from its capability to perform the motions with the required amplitudes and frequencies accurately and with a negligible deviation from an ideal sinusoidal motion. The LBR iiwa 7 R800 Kuka ${ }^{12}$ robot is used for the current work. It is a jointed-arm robot with seven axes. Every axis contains multiple sensors such as axis range sensors and torque sensors that provide signals for the robot control.

The tests have been commenced by setting the required frequency and amplitude of heaving and pitching motion through the robot control pad. Afterward, the forces in $x, y, z$ directions and the pitching, rolling and yawing moments are recorded though a software with a graphical user interface which allows saving the forces, moments, angles and position of the test case which is phase average for 100 cycles and used for post-processing and analysis. 


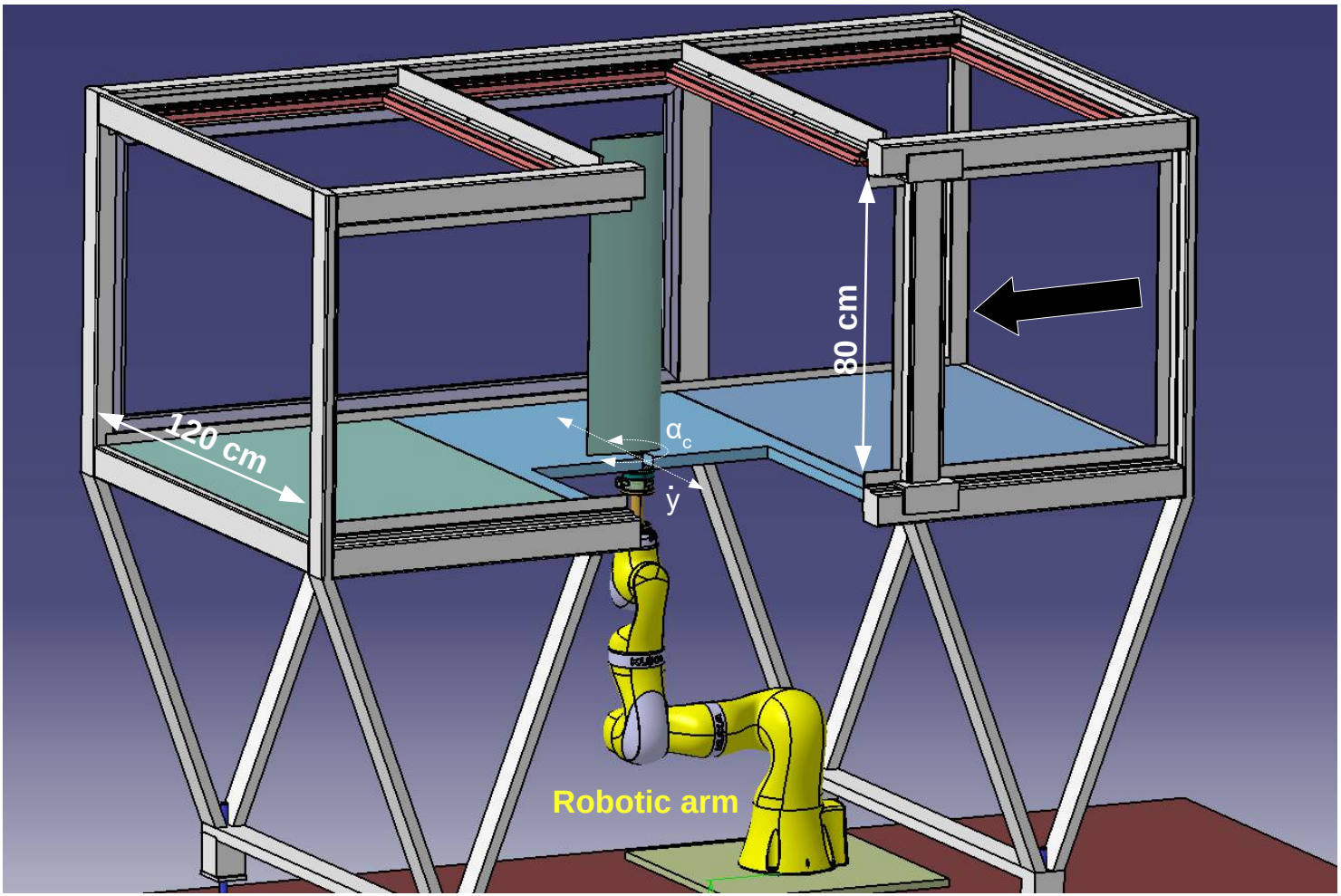

Figure 17. Test section in the low-Reynolds number wind tunnel at ISAE-SUPAERO.

\section{B. Experimental Results}

\section{Quasi-steady case}

The experimental campaign began with a quasi-steady case of pitching airfoil. The wind tunnel speed was set to $10 \mathrm{~m} / \mathrm{s}$ with a pitching frequency of $0.01 \mathrm{~Hz}$ and an amplitude of $\pm 15^{\circ}$. The objective of the first test phase was to determine the characteristics of the manufactured wing under quasi-steady aerodynamic conditions and to center the wing for a zero angle of attack and zero lift coefficient. The results shown in Figure 18 confirm that a zero lift coefficient corresponds to zero angle of attack. However, the polar reveals that the wing is not geometrically symmetric. The measurements show that the wing experiences higher drag with pitching down when compared to pitching up. The reason behind this is the fact that one surface has a slight bump due to circular spar insertion, from which we have different growth of laminar bubble between different directions of moving.

\section{Special case for constant lift coefficient}

A special case was set based on auto-stabilization flight scenario explained in section IV, for a case of pitching control with a mission to counteract the effects of wing heaving. After setting the heaving amplitude and frequency, the wing experienced a maximum translational speed of $0.5 \mathrm{~m} / \mathrm{s}$ which gave an effective maximum angle of attack of $2.85^{\circ}$ (since $V_{\infty}=10 \mathrm{~m} / \mathrm{s}$ ). The resulting pitching control for counteraction of heaving motion from eq. 9 now becomes equation 10.

$$
\alpha_{c}(t)=-2.85^{\circ} \sin (\omega t+\phi)-2.85^{\circ} a \cos (\omega t+\phi)
$$

The resulting lift coefficient during one cycle is presented in Figure 19. The results confirm that counteracting the heaving motion with only the same effective angle of pitching does not provide full suppression, especially for the frequencies with strong unsteady effects. Therefore the second part of eq. 10 serves to additionally 


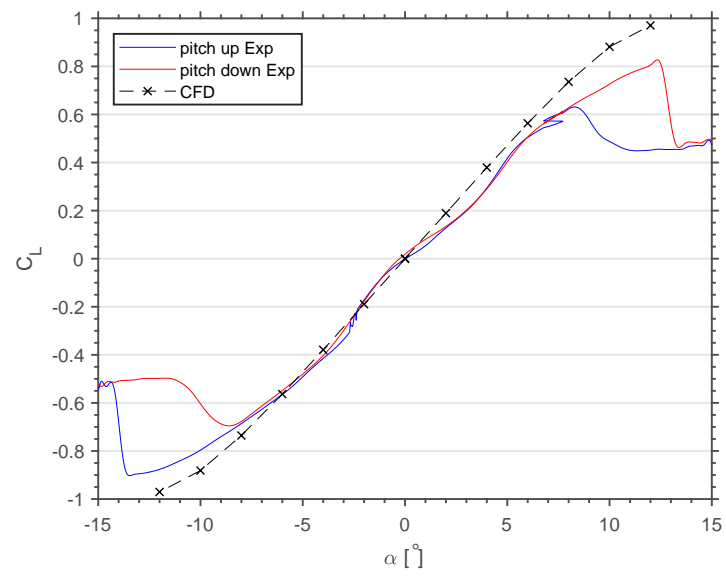

(a) Lift curve

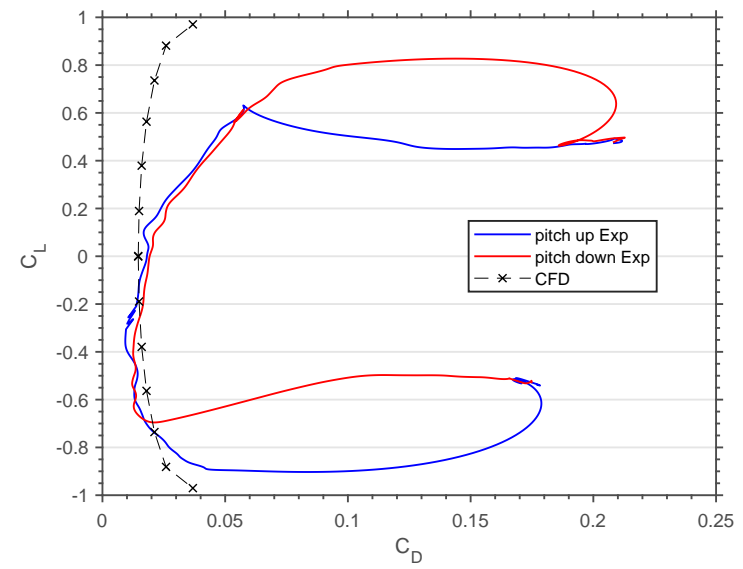

(b) Polar curve

Figure 18. Quasi-steady results for lift and drag with $R e=130,000$.

suppress the unsteady effects. The best result was achieved with the highest parameter $a=0.08$ tested, with a resulting maximum deviation for a lift coefficient of 0.01 .

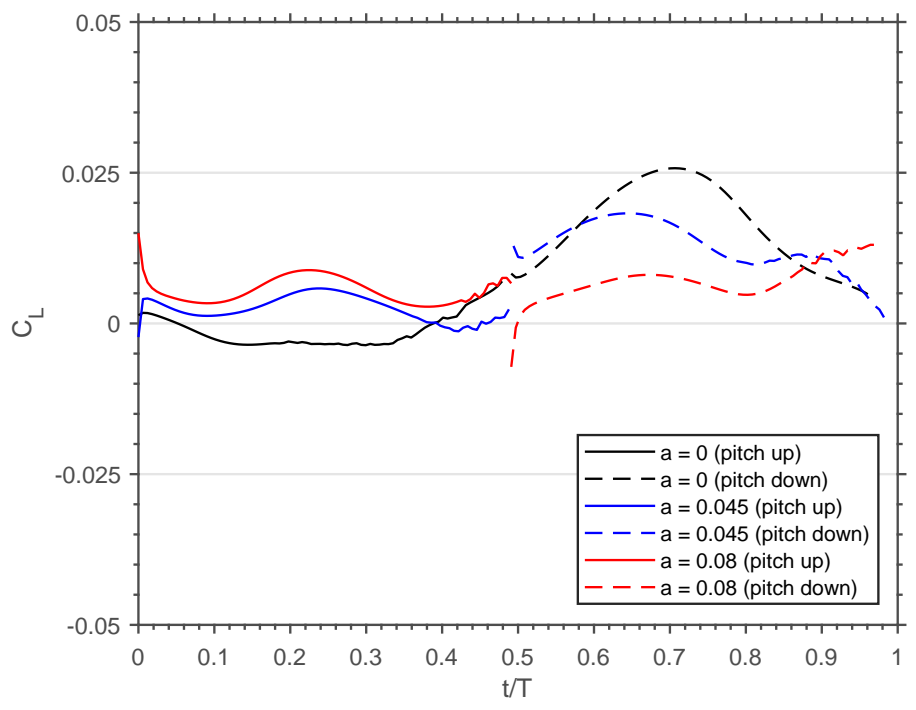

Figure 19. Constant lift coefficient $k_{g}^{*}=0.05, k_{\alpha}=-2.85^{\circ}$ and $k=0.05$.

\section{Energy-harvesting cases}

A representative energy-harvesting test case has been shown in Fig. 20. This case represents an optimal control input for a highest energy-harvesting efficiency obtained with highest reduced frequency tested, $k=0.1$. The results show a similar lift coefficient evolution between the numerical and experimental energyharvesting cycle. However, the drag coefficient evolution between the two methods has come with some considerable differences. One can notice that the drag coefficient significantly dropped for negative angles of attack of experimental results. The reason for that can be found in quasi-steady case in Fig. 18, where the drag showed non symmetrical behavior for positive and negative angle of attack due to the asymmetry of a wing.

The validation cases shown in Table 3 are that of higher gust amplitudes $\left(k_{g}=0,03 ; 0,05\right)$, since the energy harvesting effect is more significant at such high gust amplitudes. For each case, the energy harvesting 


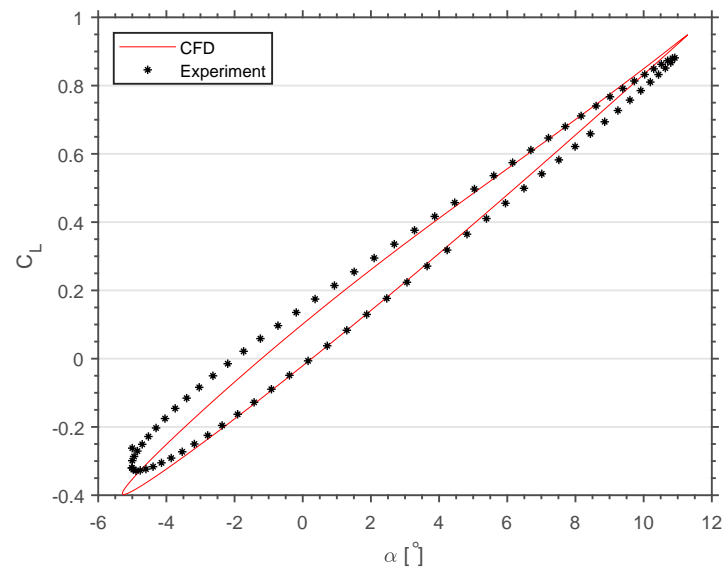

(a) Lift coefficient curve

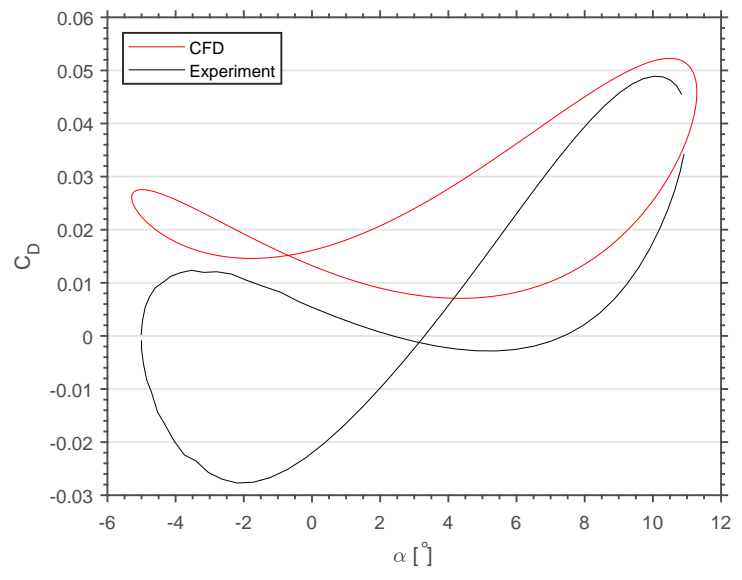

(b) Drag coefficient curve

Figure 20. Lift and drag for energy-harvesting with $k_{g}^{*}=0.05, k_{\alpha}=5.4^{\circ}, k=0.1$ and $\alpha_{0}=-3^{\circ}$.

efficiency (please see Eq. 7) is calculated at various pitch amplitudes and compared with the CFD results.

\begin{tabular}{ccccc}
\hline \hline Case & $k_{g}^{*}$ & $k$ & $\dot{y}[\mathrm{~m} / \mathrm{s}]$ & $V_{\infty}[\mathrm{m} / \mathrm{s}]$ \\
\hline a) & 0.03 & 0.025 & 0.3 & 10 \\
b) & 0.03 & 0.05 & 0.3 & 10 \\
c) & 0.05 & 0.05 & 0.5 & 10 \\
d) & 0.05 & 0.1 & 0.5 & 10 \\
\hline \hline
\end{tabular}

Table 3. Validation cases.

It can be noticed that for all the four cases, both the CFD and experimental results follow the same trend of initial growth and later decreasing efficiency. On one side, there is an error in the order of magnitude of the efficiency, and on another, there is a fair agreement between CFD and experiment in the optimal point location. For cases c) and d), it can be seen that CFD and the experiment still exhibit the same trend but at such high reduced frequencies, there is a significant shift in the optimal point location. Nevertheless, it can be concluded that the experimental investigation proved the concept of energy harvesting through vertical gust. The comparison between CFD and experiment showed the same trends of the energy harvesting efficiency variation with the pitch amplitude and a fair agreement is found for the optimal point location. The conclusions that the higher amplitude of heaving brings higher efficiency and that increasing frequency degrades energy-harvested were also confirmed in the experimental campaign.

\section{Conclusion}

In the present work, the energy harvesting through sinusoidal vertical gust is investigated through numerical simulations and wind tunnel tests. The objective was to determine the benefits of the proposed flight technique and the optimal control during the energy harvesting cycles with the aim to maximize the energy harvesting efficiency. The analysis of the gust frequency effect on the airfoil with the aerodynamic performance without control activation showed that the time delay in the peak non-dimensional force coefficients significantly increases with increasing the reduced frequency. For example, a time delay of $9 \%$ in the peak lift coefficient is found as the reduced frequency increases from 0.05 to 0.4 . Moreover, as the reduced frequency increases, the peak lift, and effective thrust coefficients decrease and consequently the peak net horizontal force coefficient increases.

Analyzing the gust amplitude effect on the airfoil aerodynamic performance without control activation showed that, the peak lift and effective thrust coefficients significantly increase, with increasing the gust 

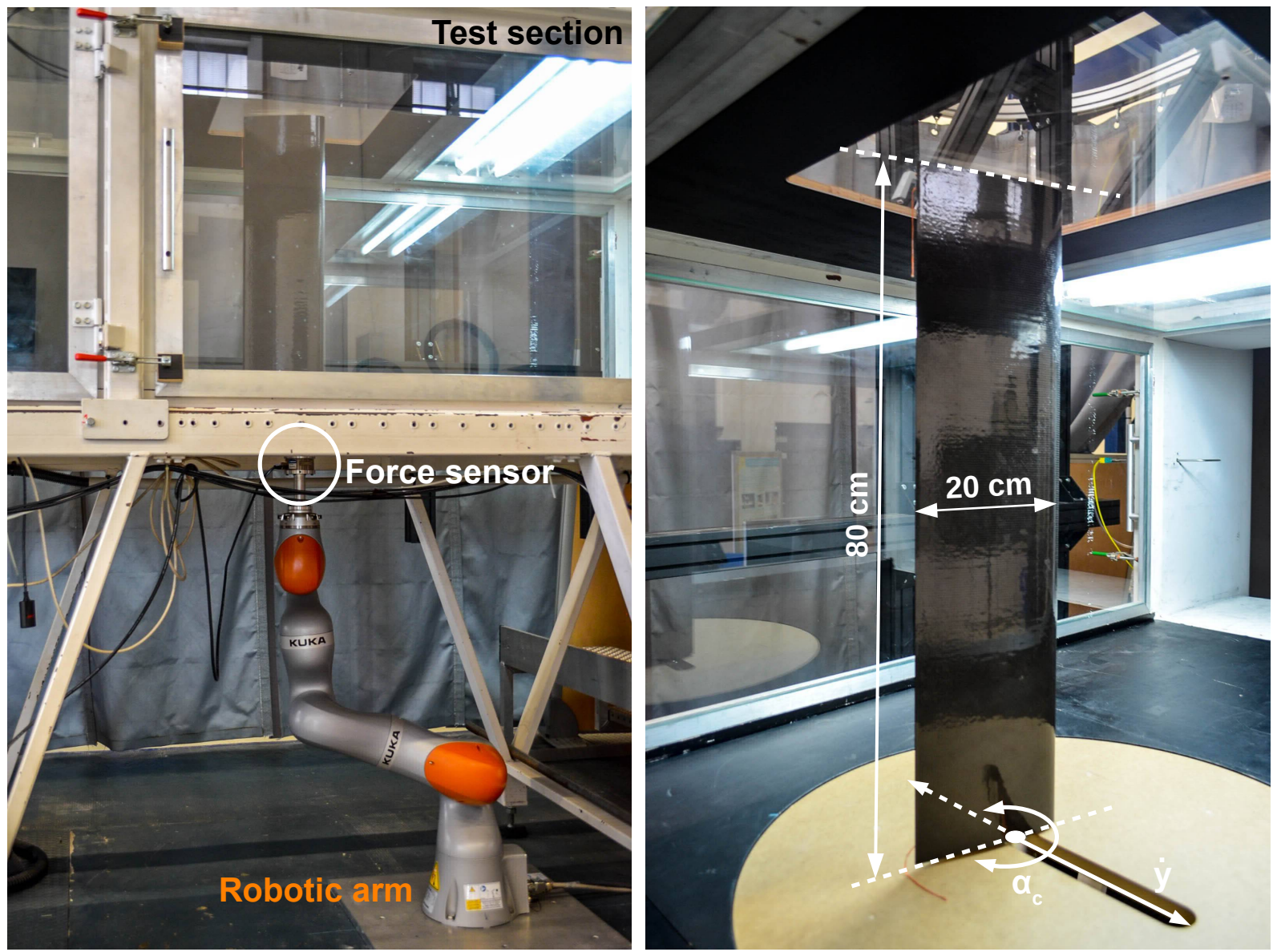

Figure 21. Experimental setup in the low-Reynolds number wind tunnel at ISAE-SUPAERO.

amplitude and consequently, the peak horizontal force coefficient decreases. For example, the peak lift coefficient is doubled as the non-dimensional gust amplitude increases from 0.035 to 0.07 . Control activation simulations showed that, for a given gust amplitude and a constant pitch input, the efficiency decreases with increasing the gust frequency. For example, at constant non dimensional gust amplitude of 0.05 and a constant pitch amplitude of $2.4^{\circ}$, the energy harvesting efficiency decreases from $36.5 \%$ to $11 \%$ as the reduced frequency increases from 0.025 to 0.2 . Moreover, for a given gust frequency and a constant pitch amplitude (control input), the energy harvesting efficiency significantly increases with increasing the gust amplitude. For example, at a constant reduced frequency of 0.1 and constant pitch amplitude of $1.4^{\circ}$, the energy harvesting efficiency increases from $1 \%$ to $13 \%$ as the non-dimensional gust amplitude increases from 0.01 to 0.05 . For an arbitrary gusty flight condition, comparison of various flight scenarios including stick fixed, constant lift coefficient and energy harvesting showed that the energy harvesting with optimal control represents a preferred flight strategy with a reduction of $61.5 \%$ in the mean horizontal force coefficient with respect to the steady flight. Finally, wind tunnel tests proved the concept of energy harvesting and exhibit the same trends of efficiency variation with pitch amplitude as that obtained through the CFD simulations. Most importantly, the experimental results confirmed the significant order of magnitude of benefits with optimal pitching control for a realistic UAV wing in conditions which could be experienced in real flight.

\section{References}

Gavrilovic, N., Bronz, M., and Moschetta, J., "Bioinspired Energy Harvesting from Atmospheric Phenomena for Small Unmanned Aerial Vehicles," Journal of Guidance, Control, and Dynamics, Vol. 43, No. 4, 2020, pp. 685-699.

${ }^{2}$ Patel, C. K. and Kroo, I., "Control Law Design for Improving UAV Performance Using Wind Turbulence," AIAA Aerospace Sciences Meeting and Exhibit, AIAA 2006-231, Reno, NV, 2006.

${ }^{3}$ Gavrilovic, N., Benard, E., Pastor, P., and Moschetta, J., "Performance Improvement of Small Unmanned Aerial Vehicles 


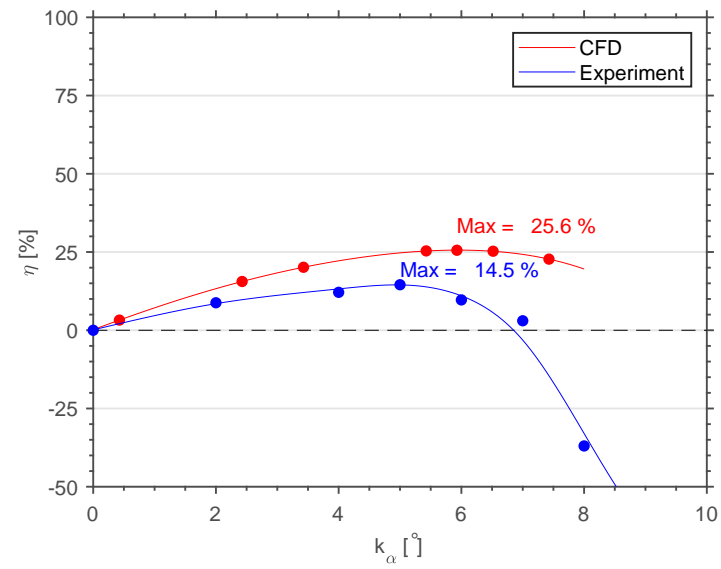

(a) $k_{g}^{*}=0.03$ and $k=0.025$

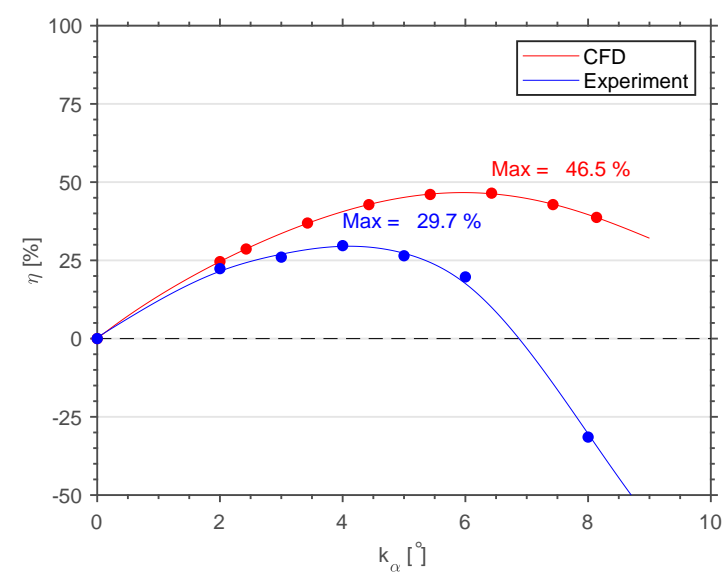

(c) $k_{g}^{*}=0.05$ and $k=0.05$

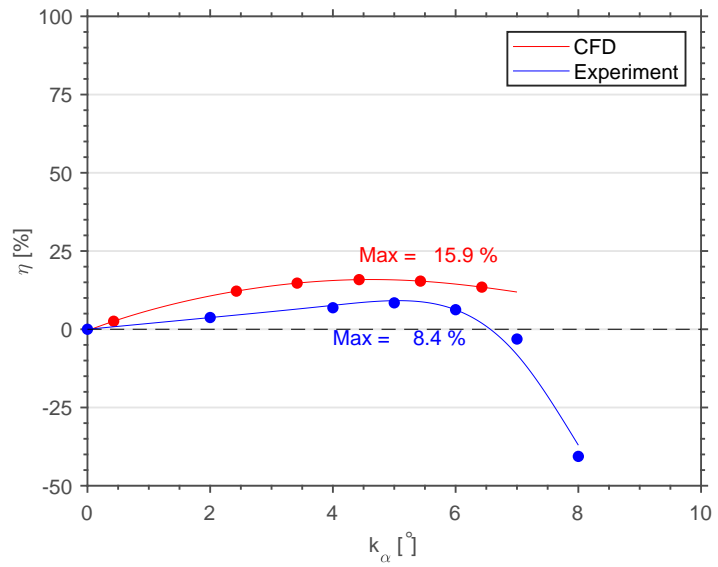

(b) $k_{g}^{*}=0.03$ and $k=0.05$

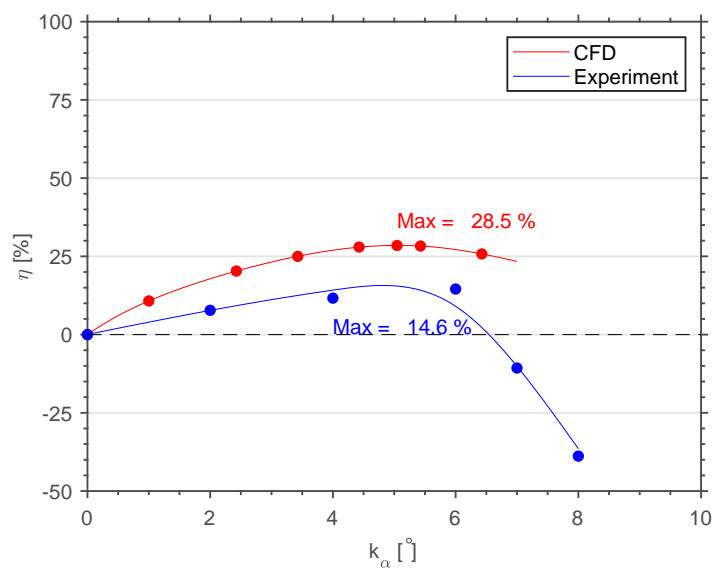

(d) $k_{g}^{*}=0.05$ and $k=0.1$

Figure 22. Comparison of the efficiency variation with the pitch amplitude between simulations and experiment.

Through Gust Energy Harvesting," Journal of Aircraft, Vol. 55, No. 2, 2018, pp. 741-754.

${ }^{4}$ Gavrilovic, N., Mohamed, A., Marino, M., Watkins, S., Moschetta, J.-M., and Benard, E., "Avian-inspired energyharvesting from atmospheric phenomena for small UAVs," Bioinspiration \& Biomimetics, Vol. 14, No. 1, 2019, pp. 1-20.

${ }^{5}$ Granlund, K., Monnier, M. O., and Wiliams, D., "Airfoil longitudinal gust response in separated vs. attached flows," Physics of Fluids, Vol. 26, No. 027103, 2014, pp. 1-14.

${ }^{6}$ Kinsey, T. and Dumas, G., "Parametric Study of an Oscillating Airfoil in a Power-Extraction Regime," AIAA Journal, Vol. 46, No. 6, 2008, pp. 1318-1330.

${ }^{7}$ Theodorsen, T., "General Theory of Aerodynamic Instability and the Mechanism of Flutter," Report 496, NACA, 1935.

${ }^{8}$ Anderson, J. M., Streitlien, K., Barrett, D. S., and Triantafyllou, M. S., "Oscillating Foils of High Propulsive Efficiency," Journal of Fluid Mechanics, Vol. 360, 1998, pp. 41-72.

${ }^{9}$ Bonnet, A., Unsteady Aerodynamics: Advanced Fluid Dynamics and Multiphysics, ISAE-SUPAERO, 2019, (in French).

${ }^{10} \mathrm{Zhu}$, Q., "Optimal frequency for flow energy harvesting of a flapping foil," Journal of Fluid Mechanics, Vol. 675, 2011, pp. $495-517$.

${ }^{11}$ Kaimal, C. and Finigan, J., Atmospheric Boundary Layer Flows, their Structure and Measurements, New York, Oxford University Press, 1994.

${ }^{12}$ KUKA, https://www.kuka.com/en-de/products/robot-systems/industrial-robots/lbr-iiwa, 2019. 